\title{
An Optimal Full Frequency Control Strategy for the Modular Multilevel Matrix Converter Based on Predictive Control
}

\author{
Boran Fan ${ }^{1}$, IEEE Student Member, Kui Wang ${ }^{1}$, IEEE Member, Pat Wheeler ${ }^{2}$, IEEE Member, Chunyang Gu ${ }^{2}$, IEEE \\ Member, Yongdong $\mathrm{Li}^{1}$, IEEE Member
}

\begin{abstract}
The modular multilevel matrix converter (M3C) is a promising topology for high-voltage high-power applications. Recent researches have proved its significant advantages for adjustable-speed motor drives compared with the back-to-back modular multilevel converter (MMC). However, the branch energy balancing in the $\mathrm{M3C}$ presents great challenge especially at critical-frequency points where the output frequency is close to zero or grid-side frequency. Generally, this balancing control depends on the appropriate injection of inner circulating currents and the common-mode voltage (CMV) whereas their values are hard to determine and optimize. In this paper, an optimization based predictive control method is proposed to calculate the required circulating currents and the CMV. The proposed method features a broad-frequency range balancing of capacitor-voltages and no reactive power in the grid side. For operation at critical-frequency points, there is no increase on branch voltage stresses and limited increase on branch current stresses. A downscaled M3C system with 27 cells is designed and experiment results with the R-L load and induction motor load are presented to verify the proposed control method.
\end{abstract}

Index Terms - Modular multilevel matrix converter (M3C), triple-star bridge cells (TSBC) converter, voltage balancing control, low frequency, equal frequency, medium-voltage high-power ASD.

\section{INTRODUCTION}

The modular multilevel matrix converter (M3C) or triple-star bridge cells (TSBC) converter [1], shown in Fig. 1 (a), can be used to connect two three-phase electrical systems (input side and output side systems) using nine active branches. Each branch consists of a cascaded connection of full-bridge cells and a branch inductor. This topology was first proposed by R. Erickson and O. Al-Naseem in 2001 [2]. In common with other members of the modular multilevel cascade converter family [1], the M3C can easily reach high voltage ratings as well as significantly reduce voltage harmonics and electromagnetic interference. In addition, the modular structure makes it easier to accomplish construction, maintenance and thermal designs.

Initially, the $\mathrm{M} 3 \mathrm{C}$ topology was presented and researched without branch inductors. Space vector modulation (SVM) based capacitor-voltage balancing methods are proposed in [3]-[5].

Manuscript submitted April 26, 2017; revised July 26, 2017; accepted September 16, 2017.

This work was supported by the Education Development Program of Delta Environmental \& Educational Foundation (Grant No. DREK2015001).

Color versions of one or more of the figures in this paper are available online at http://ieeexplore.iee.org.

Boran Fan, Kui Wang and Yongdong Li are with the State Key Laboratory of Power System, Department of Electrical Engineering, Tsinghua University, Beijing 100084, China. (e-mail: fbr13@mails.tsinghua.edu.cn; wangkui@tsinghua.edu.cn; liyd@tsinghua.edu.cn). Pat Wheeler and Chunyang Gu are with Department of Electrical and Electronic Engineering, University of Nottingham, Nottingham NG7 2RD, United Kingdom. (e-mail: Pat.Wheeler@nottingham.ac.uk; chunyang.gu@nottingham.ac.uk).

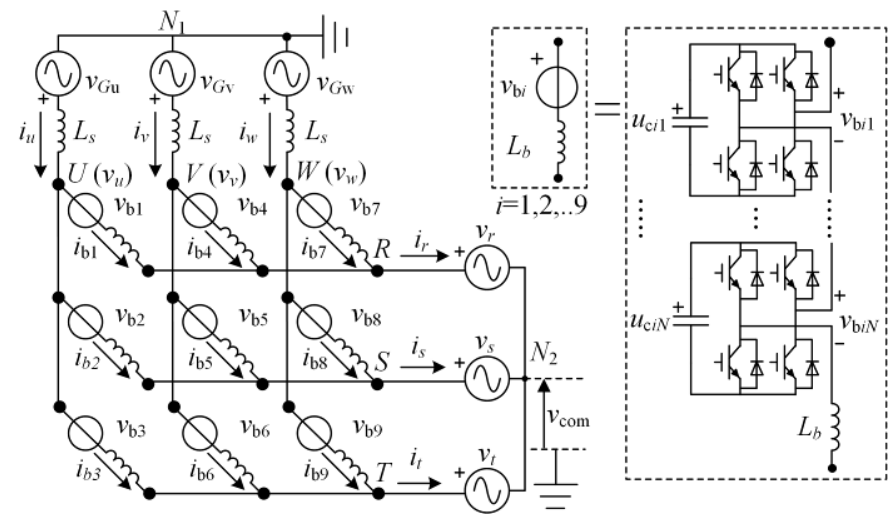

(a)

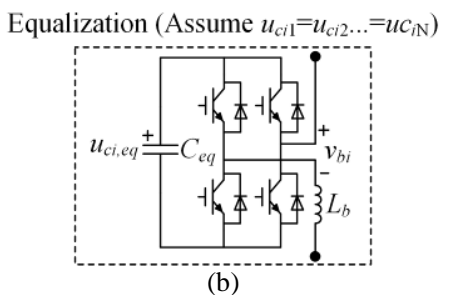

Fig. 1. (a) Circuit configuration of the M3C, (b) Branch equalization.

However, the large number of redundant space vectors in M3C makes these methods difficult to implement when more than one cell is used in each branch. Moreover, since each branch works as a controlled voltage source, to avoid parallel connection of cell capacitors, normally not all branches conduct current at the same time. This unbalanced current distribution results in a higher branch current stress. In 2009, C. Oates presented the M3C structure with branch inductors and made branches work as controlled current sources [6]. The significant benefit brought by this change is the possibility to employ a carrier-based pulse-width modulation (PWM) method and hence the control complexity becomes relatively independent of cell numbers. On this basis, in 2012, F. Kammerer et al. [7] and W. Kawamura et al. [8] proposed an effective approach to realize a decoupled control on input currents, output currents and inner circulating currents in the $\mathrm{M} 3 \mathrm{C}$ by the application of a 'double $\alpha \beta 0$ transformation' [9], [10].

The modular multilevel converter (MMC) is another promising modular multilevel cascade converter, which has been practically used in high-voltage direct current transmissions. However, MMC suffers large capacitor-voltage fluctuation at low-frequency operation [11]-[13]. Compared with the back-to-back MMC configuration, the capacitor-voltage fluctuation in $\mathrm{M} 3 \mathrm{C}$ is significantly reduced at low-frequency operation [14]-[16] because of the frequency coupling of the two three-phase systems. This advantage makes M3C more suitable for high-voltage, high-power adjustable speed drive (ASD) applications such as offshore wind-power generations [17] and full-electric marine propulsion systems 
[18]. The application of $\mathrm{M} 3 \mathrm{C}$ in low-frequency $\mathrm{AC}$ transmission (LFAC) systems [19], [20] is also very promising where $\mathrm{M} 3 \mathrm{C}$ can substitute the thyristor-based cycloconverter to reduce current harmonics and improve the power factor.

However, the frequency coupling of the input and output system in M3C also causes large capacitor-voltage fluctuation when the input and output frequencies get closer [21]. Meanwhile, even though the capacitor-voltage fluctuation in $\mathrm{M} 3 \mathrm{C}$ at low-frequency operation is far better than MMC, there still exists a double output-frequency component in the branch power and the magnitude is proportional to the output power. Therefore, when large reactive/active power is deployed at low-frequency operation, large capacitor-voltage fluctuations still exists in $\mathrm{M} 3 \mathrm{C}$. The control on these two critical-frequency points (i.e. equal-frequency and low-frequency operations) should be carefully designed to realize a stable application of $\mathrm{M} 3 \mathrm{C}$ at broad-frequency range.

To stabilize the M3C operation at low-frequency point, [22] presented a method by injecting circulating currents, referred as "instantaneous power mode". The issue of this method is that the capacitor-voltage fluctuation is amplified when the output frequency is third of the input frequency. To solve the problem, in [8], the mode of the injected circulating currents was improved by including the adjustment on instantaneous reactive power in each branch.

To stabilize the M3C operation at equal-frequency point, [23] proposed a method by injecting circulating currents and applying reactive power at the input-side (supply-side). The main problem of this method is that the branch current stresses can reach two times higher than the condition when no circulating current is injected. Moreover, the reactive power applied at the supply-side is the same as the output-side, which seriously decrease the supply power quality especially when driving an induction motor. The ideas described in [24] use an adjustment in the motor magnetizing current to ensure that the input and output side share the same voltage magnitude at equal-frequency point. This method helps to achieve lower branch current stresses but in practice, the condition of same input and output voltage magnitude is difficult to hold. Moreover, the method in [24] also needs to apply the same reactive power in [23] at the input side. To reduce the reactive power of the supply-side, [21] proposed a method by only using circulating currents to realize the equal-frequency operation. However, when the input and output voltage magnitudes get closer, part of the load-side reactive power needs to be gradually applied at the supply-side. The work in [25] and [26] introduced both common-mode voltage (CMV) and circulating currents for the balancing control at equal-frequency point, a similar technique to the mitigation control of the MMC at low-speed range [11]-[13]. However, the references for the CMV and circulating currents are difficult to design and it requires an extra margin on branch voltage stresses for the CMV injection.

In sum, the existing methods use different forms of circulating current/CMV injection or supply-side reactive power at different operating frequency, hereby a switching over between these methods is required as operating frequency changes. Besides, the optimization of CMV and circulating currents has not been fully considered yet. The aim of this paper is to present a capacitor-voltage balancing method in the full frequency range for the $\mathrm{M} 3 \mathrm{C}$ by optimal $\mathrm{CMV}$ and circulating current injection. The proposed control can achieve unity factor at supply-side at any operation frequency and require no increase on branch voltage stresses and limited increase on branch current stresses for critical-frequency operations. Section II analyzes the branch power and capacitor-voltage fluctuation without injection of circulating current and CMV. Section III explains the proposed method of optimized CMV and circulating current injection in detail. Section IV presents the overall control for the $\mathrm{M} 3 \mathrm{C}$ based on the optimal method. Experimental results are presented in Section V to validate the proposed control method, and Section VI concludes the paper.

\section{BASIC THEORY OF THE M3C}

\section{A. M3C modeling and the basic branch currents}

Fig. 1 (a) shows the detailed circuit configuration of the $\mathrm{M} 3 \mathrm{C}$, which connects two three-phase systems with nine branches. Each branch consists of a branch inductor $L_{b}$ and a string of cascaded full-bridges. In this paper, the input and output three-phase systems are denoted as 'UVW' and 'RST'. Applying Kirchhoff's Voltage Law to the nine branches:

$$
\begin{aligned}
& {\left[\begin{array}{lll}
v_{u}-v_{r} & v_{u}-v_{s} & v_{u}-v_{t} \\
v_{v}-v_{r} & v_{v}-v_{s} & v_{v}-v_{t} \\
v_{w}-v_{r} & v_{w}-v_{s} & v_{w}-v_{t}
\end{array}\right]-v_{c o m} \mathbf{I}_{3 \times 3}} \\
& L_{\mathrm{b}} \frac{d}{d t}\left[\begin{array}{lll}
i_{b 1} & i_{b 2} & i_{b 3} \\
i_{b 4} & i_{b 5} & i_{b 6} \\
i_{b 7} & i_{b 8} & i_{b 9}
\end{array}\right]+\left[\begin{array}{ccc}
v_{b 1} & v_{b 2} & v_{b 3} \\
v_{b 4} & v_{b 5} & v_{b 6} \\
v_{b 7} & v_{b 8} & v_{b 9}
\end{array}\right], \mathbf{I}_{3 \times 3}=\left[\begin{array}{ccc}
1 & 1 & 1 \\
1 & 1 & 1 \\
1 & 1 & 1
\end{array}\right] .
\end{aligned}
$$

In (1), $v_{u}, v_{v}, v_{w}$ are input-side voltages and $v_{r}, v_{s}, v_{t}$ are output-side voltages. And $i_{b i}(i=1,2, . .9)$ represent branch currents and $v_{b i}(i=1,2, . .9)$ are the output voltages of cascaded full-bridges. The neutral point $\mathrm{N} 1$ is referred as the zero potential and the CMV (denoted as $v_{\text {com }}$ ) is defined as the voltage difference between point $\mathrm{N} 2$ and $\mathrm{N} 1$. In recent literatures, a so-called double $\alpha \beta 0$ transformation is designed to realize a decoupled current control for the input, output and inner circulating currents [7]-[10], [27]. The definition of the double $\alpha \beta 0$ transformation $\mathbf{T}_{\text {Dual- } \alpha \beta}$ is in (2), which is a linear transformation performed on a $3 \times 3$ matrix $\mathrm{M}_{3 * 3}$.

$$
\mathbf{T}_{\mathbf{D} \alpha \mathbf{\beta}}\left(\mathbf{M}_{3 * 3}\right)=\mathbf{T}_{\alpha \boldsymbol{\beta}} \cdot \mathbf{M}_{3 * 3} \cdot \mathbf{T}_{\alpha \boldsymbol{\beta}}{ }^{\mathbf{T}}, \mathbf{T}_{\alpha \boldsymbol{\beta}}=\frac{1}{3}\left[\begin{array}{ccc}
2 & -1 & -1 \\
0 & \sqrt{3} & -\sqrt{3} \\
1 & 1 & 1
\end{array}\right]
$$

Applying this double $\alpha \beta 0$ transformation to (1):

$$
\begin{aligned}
& {\left[\begin{array}{ccc}
0 & 0 & v_{\alpha}^{u v w} \\
0 & 0 & v_{\beta}^{u v w} \\
-v_{\alpha}^{r s t} & -v_{\beta}^{r s t} & -v_{c o m}
\end{array}\right]=L_{\mathrm{b}} \frac{d}{d t}\left[\begin{array}{ccc}
i_{\alpha \alpha} & i_{\alpha \beta} & i_{\alpha}^{u v w} / 3 \\
i_{\beta \alpha} & i_{\beta \beta} & i_{\beta}^{u v w} / 3 \\
i_{\alpha}^{r s t} / 3 & i_{\beta}^{r s t} / 3 & 0
\end{array}\right]+} \\
& {\left[\mathbf{v}_{\mathbf{b}}\right]_{\mathbf{D} \alpha \beta},\left[\mathbf{v}_{\mathbf{b}}\right]_{\mathbf{D} \alpha \beta}=\left[\begin{array}{llll}
v_{\alpha \alpha} & v_{\alpha \beta} & v_{\alpha 0} \\
v_{\beta \alpha} & v_{\beta \beta} & v_{\beta 0} \\
v_{0 \alpha} & v_{0 \beta} & v_{00}
\end{array}\right] .}
\end{aligned}
$$


In (3), $v_{\alpha}^{u v w}, v_{\beta}^{u v w}$ and $i_{\alpha}^{u v w}, i_{\beta}^{u v w}$ are the input voltages and input currents $\left(i_{u}, i_{v}, i_{w}\right)$ on the $\alpha \beta$ reference frames. $v_{\alpha}^{r s t}, v_{\beta}^{r s t}$ and $i_{\alpha}^{r s t}, i_{\beta}^{r s t}$ are the output voltages and output currents $\left(i_{r}, i_{s}\right.$, $i_{t}$ ) on the $\alpha \beta$ reference frames. $i_{\alpha \alpha}, i_{\alpha \beta}, i_{\beta \alpha}, i_{\beta \beta}$ represent the four inner circulating currents. These four currents are independent of input and output currents. The nine elements in matrix $\left[\mathbf{v}_{\mathbf{b}}\right]_{\mathbf{D}-\mathbf{\alpha}, \mathbf{\beta}}$ are the output voltages of the nine cascaded full-bridges on the double $\alpha \beta$ reference frames. According to (3), decoupled control on input, output and inner circulating currents can be performed by adjusting the value of matrix [ $\left.\mathbf{v}_{\mathbf{b}}\right]_{\mathbf{D} \alpha \beta}$. In (3), $v_{\text {com }}$ is the CMV as shown in Fig. 1 (a). The value of $v_{\text {com }}$ can be controlled by $v_{00}$ in $\left[\mathbf{v}_{\mathbf{b}}\right]_{\text {Da }}$. If set the value of circulating currents $i_{\alpha \alpha}, i_{\alpha \beta}, i_{\beta \alpha}$ and $i_{\beta \beta}$ as zero and apply an inverse transformation of $\mathbf{T}_{\text {Dual-aß }}$ on branch currents, the nine branch currents $i_{b 0, i}(i=1,2, . .9)$, shown in (4), consist of $1 / 3$ of the $x$-phase $(x=u, v, w)$ input side current and $1 / 3$ of the $y$-phase $(y=r, s, t)$ output side current. In this paper, the branch currents in (4) are defined as the 'basic branch currents'.

$$
\left[\begin{array}{lll}
i_{b 0,1} & i_{b 0,2} & i_{b 0,3} \\
i_{b 0,4} & i_{b 0,5} & i_{b 0,6} \\
i_{b 0,7} & i_{b 0,8} & i_{b 0,9}
\end{array}\right]=\frac{1}{3}\left[\begin{array}{lll}
i_{u}+i_{r} & i_{u}+i_{s} & i_{u}+i_{t} \\
i_{v}+i_{r} & i_{v}+i_{s} & i_{v}+i_{t} \\
i_{w}+i_{r} & i_{w}+i_{s} & i_{w}+i_{t}
\end{array}\right]
$$

\section{B. Voltage fluctuation with basic branch currents}

In the following analysis, the input and output systems are assumed three-phase balanced. The input side is connected with AC supply through AC inductor $L_{s}$. For the input system, phase voltage $v_{u}$ and current $i_{u}$ are defined in (5). Neglecting AC inductor $L_{s}, v_{u}$ is defined in phase with $i_{u}$ to ensure no reactive power at the AC grid (i.e. unity power factor). When $f_{1}>0, v_{u} / i_{u}$ $\left(v_{v} / i_{v}\right)$ is 120 degrees leading $v_{v} / i_{v}\left(v_{w} / i_{w}\right)$. For the output system, phase voltage $v_{r}$ and current $i_{r}$ are defined in (6). When $t=0, v_{r}$ is $\theta$ degrees leading $v_{u}$. The power factor angle at the output system is $\varphi$. When $f_{2}>0, v_{r} / i_{r}\left(v_{s} / i_{s}\right)$ is 120 degrees leading $v_{s} / i_{s}$ $\left(v_{t} / i_{t}\right)$

$$
\begin{gathered}
v_{u}=\hat{v}_{m 1} \cos \left(2 \pi f_{1} \cdot t\right), i_{u}=\hat{i}_{m 1} \cos \left(2 \pi f_{1} \cdot t\right) . \\
v_{r}=\hat{v}_{m 2} \cos \left(2 \pi f_{2} \cdot t+\theta\right), i_{r}=\hat{i}_{m 2} \cos \left(2 \pi f_{2} \cdot t+\theta-\varphi\right) .
\end{gathered}
$$

With the applying of basic branch currents in (4) and without the using of CMV (i.e. $v_{c o m}=0$ ), the basic branch power $p_{b 0, i}$ $(i=1,2, . .9)$ is calculated in (7).

$$
p_{b 0, i}=i_{b 0, i}\left(v_{x}-v_{y}\right)=p_{b i}^{d c}+\left.\sum p_{b i}^{f}\right|_{f=2 f_{1}, 2 f_{2}, f_{1}-f_{2}, f_{1}+f_{2}} .
$$

Assuming $f_{1} \neq \pm f_{2}$ and $f_{1}, f_{2} \neq 0$, the basic branch power $p_{b 0 i}$ consists of frequency components at $0, f_{1}-f_{2}, f_{1}+f_{2}, 2 f_{1}$ and $2 f_{2}$. Obviously, $p_{b i}^{d c}$ must be set as zero otherwise the branch energy will become unstable. It can be proved that the value of the dc power component $p_{b i}^{d c}$ on nine branches are the same $p_{b 1}^{d c}=p_{b 2}^{d c} \ldots=p_{b 9}^{d c}$. Therefore $p_{b i}^{d c}(i=1,2, . .9)$ could be fully eliminated by balancing the active power between the input and output systems. With $p_{b 1}^{d c}=\ldots=p_{b 9}^{d c}=0$, the capacitor voltage fluctuation ratio $\pm \eta \%$ is calculated in (8), where $\hat{p}_{2 f_{1}}, \hat{p}_{2 f_{2}}, \hat{p}_{f_{1}-f_{2}}$ and $\hat{p}_{f_{1}+f_{2}}$ are branch power magnitudes at the four different frequency, $N$ is the number of cascaded full-bridges in each branch and $U_{C 0}^{*}$ is the reference value of the cell capacitor voltage. It can be verified that the value of $\hat{p}_{2 f_{1}}, \hat{p}_{2 f_{2}}, \hat{p}_{f_{1}-f_{2}}$ and $\hat{p}_{f_{1}+f_{2}}$ are the same for all nine branches.

$$
\begin{aligned}
& \eta \%=\frac{0.5 \Delta E_{p-p}}{N C U_{C 0}^{* 2}}, \\
& \Delta E_{p-p}=\left|\frac{\hat{p}_{2 f_{1}}}{2 \pi f_{1}}\right|+\left|\frac{\hat{p}_{2 f_{2}}}{2 \pi f_{2}}\right|+\left|\frac{\hat{p}_{f_{1}-f_{2}}}{\pi f_{1}-\pi f_{2}}\right|+\left|\frac{\hat{p}_{f_{1}+f_{2}}}{\pi f_{1}+\pi f_{2}}\right|
\end{aligned}
$$

Fig. 2 (a) is the capacitor-voltage fluctuation ratio when a specific M3C (parameters are presented in Section V) is connected with an R-L load and the magnitude of the output voltage magnitude $\hat{v}_{m 2}$ remains constant when the output frequency $f_{2}$ changes. In Fig. 2 (a), the voltage fluctuation becomes worse at critical-frequency points $\left(f_{2}=0\right.$ and $\left.f_{2}=f_{1}\right)$. Fig. 2 (b) is the voltage fluctuation ratio when the $\mathrm{M} 3 \mathrm{C}$ is connected with an induction motor loaded at a constant torque. The red line in Fig. 2 (b) shows the relationship between the actual output frequency $f_{2}$ and the motor speed. In Fig. 2 (b), the voltage fluctuation becomes worse at low-frequency points $\left(f_{2}=0\right)$. Due to the slip frequency and small output power, when motor speed gets close to zero, the capacitor voltage fluctuation in Fig. 2(b) does not dramatically aggravate. Compared with MMC, Fig.2 (b) proves the advantages of M3C for low-frequency constant-torque drive application, as the capacitor-voltage fluctuation is relatively limited at low speed of motor.

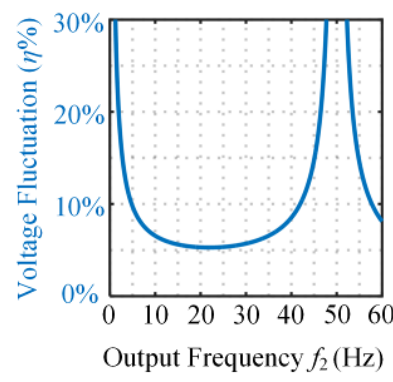

(a)

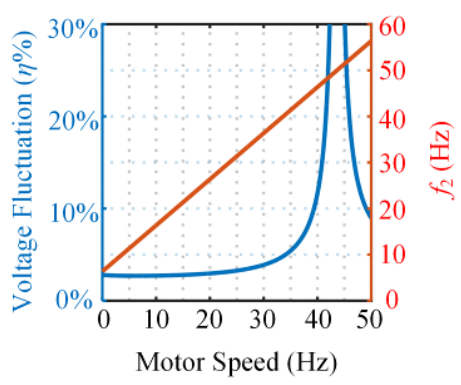

(b)
Fig. 2. Capacitor-voltage fluctuation (input frequency $f_{1}=50 \mathrm{~Hz}$ ). (a) R-L load (output voltage magnitude is constant), (b) Constant-torque induction motor load.

Fig. 2 shows the problem when operating M3C around critical-frequency points. The large capacitor-voltage fluctuation is caused by certain ac branch-power components in (7) progressively becoming a low-frequency/dc power near/at critical-frequency points. For instance, when $f_{2}=f_{1}$, branch power component $p_{b i}^{\omega=\omega 1-\omega 2}(i=1,2, . .9)$ becomes a dc power, denoted as transferred dc components $\Delta p_{b i}^{d c}$. Unlike $p_{b i}^{d c}$ in (7), these transferred dc components $\Delta p_{b i}^{d c}$ have different values on nine branches, so it is impossible to eliminate all of them by 
balancing the active power between the input and output systems. When $f_{2}=f_{1}$, these transferred dc powers on nine branches satisfy $\Delta p_{b 1}^{d c}=\Delta p_{b 5}^{d c}=\Delta p_{b 9}^{d c}, \quad \Delta p_{b 2}^{d c}=\Delta p_{b 6}^{d c}=\Delta p_{b 7}^{d c} \quad$ and $\Delta p_{b 3}^{d c}=\Delta p_{b 4}^{d c}=\Delta p_{b 8}^{d c}$ so branch-balancing control needs to balance branch energies among these three branch groups. Using the direction definition of capacitor voltages in [7], branch balancing control can be visualized as balancing along 'positive diagonals' as shown in Fig.3. Similarly, when $f_{1}=-f_{2}, f_{1}=0$ or $f_{2}=0$, the analysis is shown in Tab. 1 and Fig. 3 .

TABLE I

Additional DC Branch Power

\begin{tabular}{llll}
\hline \hline & $\Delta p_{b i}^{d c}$ & $(i, j, k) s . t . \Delta p_{b i}^{d c}=\Delta p_{b j}^{d c}=\Delta p_{b k}^{d c}$ & Voltage Direction \\
\hline$f_{1}=0$ & $p_{b i}^{\omega=2 \omega_{1}}$ & $(1,2,3)(4,5,6)(7,8,9)$ & Horizontal \\
\hline$f_{2}=0$ & $p_{b i}^{\omega=2 \omega_{2}}$ & $(1,4,7)(2,5,8)(3,6,9)$ & Vertical \\
\hline$f_{2}=f_{1}$ & $p_{b i}^{\omega=\omega_{1}+\omega_{2}}$ & $(1,5,9)(2,6,7)(3,4,8)$ & Positive diagonal \\
\hline$f_{2}=-f_{1}$ & $p_{b i}^{\omega=\omega_{1}-\omega_{2}}$ & $(1,6,8)(2,4,9)(3,5,7)$ & Negative diagonal \\
\hline
\end{tabular}

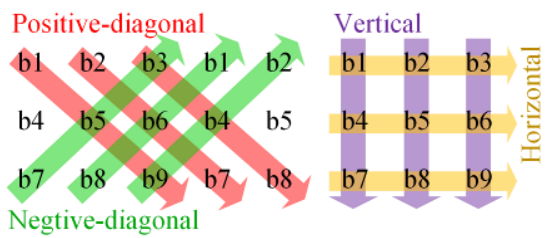

Fig. 3. Direction definitions of capacitor voltages.

The above analysis explains the different requirements of balancing control at different frequencies. The aim of this paper is to present a unified control strategy by the optimal injection of CMV and circulating currents.

\section{OPtimization BASEd PREDictive Control Method}

In the $\mathrm{M} 3 \mathrm{C}$, the cell capacitor-voltage balancing in each branch can be achieved by many well-constructed methods like capacitor voltage sorting based on phase-disposition carrier pulse-width modulation (PWM) (PD-PWM) [11] or reference voltage adjustment for each full-bridge based on phase-shift carrier PWM (PS-PWM) [28]. As a result, for simplicity, the following analysis assumes capacitor-voltages are balanced in each branch. Therefore, for each branch, the cascaded full-bridges can be equalized as a single full-bridge as shown in Fig. 1 (b). The equivalent cell capacitor reference voltage and equivalent capacitance are $U_{C 0, e q}^{*}=N \cdot U_{C 0}^{*}$ and $C_{e q}=C / N$. The equivalent capacitor-voltage and the equivalent branch output voltage are $u_{c i, e q}=\sum_{j=1,2, \ldots N} u_{c i j}$ and $v_{b i}=\sum_{j=1,2, \ldots N} v_{b i j}(i=1,2, . .9)$ respectively.

\section{A. Optimized CMV injection}

The CMV and inner circulating currents are the two types of control degrees in the M3C. In this part, a CMV design method is proposed to help realizing the capacitor-voltage balancing. Neglecting the branch inductor $L_{b}$, output voltages of nine cascaded full-bridges are the same as the phase voltage differences between the input and output system:

$$
\left[\begin{array}{lll}
v_{b 1, \text { ref }} & v_{b 2, \text { ref }} & v_{b 3, \text { ref }} \\
v_{b 4, \text { ref }} & v_{b 5, \text { ref }} & v_{b 6, \text { ref }} \\
v_{b 7, \text { ref }} & v_{b 8, \text { ref }} & v_{b 9, \text { ref }}
\end{array}\right] \approx\left[\begin{array}{lll}
v_{u}-v_{r} & v_{u}-v_{s} & v_{u}-v_{t} \\
v_{v}-v_{r} & v_{v}-v_{s} & v_{v}-v_{t} \\
v_{w}-v_{r} & v_{w}-v_{s} & v_{w}-v_{t}
\end{array}\right]
$$

In practice, considering the capacitor-voltage fluctuation of $\pm \eta \%$, the equivalent capacitor reference voltage $U_{C 0, e q}^{*}$ should satisfy (10) to withstand the maximum voltage difference between the input and output systems. Here $\hat{v}_{m 1}^{\max }$ and $\hat{v}_{m 2}^{\max }$ refer to the maximum voltage magnitudes of the input and output three-phase systems.

$$
U_{C 0, e q}^{*}=\left(\hat{v}_{m 1}^{\max }+\hat{v}_{m 2}^{\max }\right) /(1-\eta \%)
$$

According to (1), with the injection of CMV, the output voltage references of cascaded full-bridges are in (11).

$$
\begin{aligned}
& v_{b i, r e f, c o m}=v_{b i, r e f}-v_{c o m}, \\
& -\left(\hat{v}_{m 1}^{\max }+\hat{v}_{m 2}^{\max }\right) \leq v_{b i, r e f, c o m} \leq\left(\hat{v}_{m 1}^{\max }+\hat{v}_{m 2}^{\max }\right), i=1,2, . .9 .
\end{aligned}
$$

Dividing (11) by $U_{C 0, e q}^{*}$, the per-unit (p.u.) branch output voltage references are written in (12) where $v_{b i, \text { ref }}^{p u}$ and $v_{c o m}^{p u}$ are the p.u. value of the phase voltage differences and the CMV.

$$
\begin{aligned}
& v_{b i, r e f, c o m}^{p u}=v_{b i, r e f}^{p u}-v_{c o m}^{p u}, \\
& -1+\eta \% \leq v_{b i, r e f, c o m}^{p u} \leq 1-\eta \%, i=1,2, . .9 .
\end{aligned}
$$

Obviously, to avoid over-modulation, voltage references $v_{b i, r e f, c o m}^{p u}$ must be in the range of $-1+\eta \%$ to $1-\eta \%$. Accordingly, the CMV must satisfy (13) to (15) where $v_{x}^{p u}=v_{x} / U_{C 0, e q}^{*}(x=u, v, w)$ is the p.u. value of the input-side voltage, $v_{y}^{p u}=v_{y} / U_{C 0, e q}^{*}(y=r, s, t)$ is the p.u. value of the output-side voltage and $\xi(\xi \in[0,1])$ is an adjustable factor to limit the magnitude of the injected CMV.

$$
v_{\text {com }}^{p u} \in\left[v_{\text {com_min }}^{p u}, v_{\text {com_max }}^{p u}\right]
$$

where

$$
\begin{aligned}
& v_{\text {com_min }}^{p u}=\xi \cdot\left(\max \left(v_{b i, r e f} \mid i=1,2, \ldots 9\right)-(1-\eta \%)\right) \\
& =\xi \cdot\left(\max \left(v_{u}^{p u}, v_{v}^{p u}, v_{w}^{p u}\right)-(1-\eta \%)\right)-\xi \cdot \min \left(v_{r}^{p u}, v_{s}^{p u}, v_{t}^{p u}\right), \\
& v_{\text {com_max }}^{p u}=\xi \cdot\left(\min \left(v_{b i, r e f} \mid i=1,2, \ldots 9\right)+(1-\eta \%)\right) \\
& =\xi \cdot\left(\min \left(v_{u}^{p u}, v_{v}^{p u}, v_{w}^{p u}\right)+(1-\eta \%)\right)-\xi \cdot \max \left(v_{r}^{p u}, v_{s}^{p u}, v_{t}^{p u}\right) .
\end{aligned}
$$

According to (9) and (11), when the input and output voltage magnitudes satisfy $\hat{v}_{m 1} \leq \hat{v}_{m 1}^{\max }$ and $\hat{v}_{m 2} \leq \hat{v}_{m 2}^{\max }$, obviously none CMV injection ( $v_{c o m}^{p u}=0$ ) will not cause over-modulation so the 
minimum and maximum value of the CMV satisfies $v_{\text {com_min }}^{p u} \leq 0 \leq v_{\text {com_max }}^{p u}$. Taking conditions of ' $\hat{v}_{m 1}=\hat{v}_{m 1}^{\max }$, $\hat{v}_{m 2}=\hat{v}_{m 2}^{\max }, \xi=1, f_{1}=f_{2}, \theta=0$ ' and ' $\hat{v}_{m 1}=\hat{v}_{m 1}^{\max }, \hat{v}_{m 2}=\hat{v}_{m 2}^{\max }, \xi=1$, $f_{1}=f_{2}, \theta=180^{\circ}$ ' as examples, the applicable range of the CMV is shown in light blue filling in Fig. 4 (a) and Fig. 4 (b).

The applicable CMV range presented in (13) is continuous. To perform the optimization algorithm, the range in (13) are equally discretized into $N_{\text {com }}+1$ applicable CMV values as

$$
v_{\text {com }}^{p u, j}=v_{\text {com_min }}^{p u}+\left(v_{\text {com_max }}^{p u}-v_{\text {com_min }}^{p u}\right) \cdot j / N_{\text {com }}, j=0,1, . . N_{\text {com }} .
$$

At control moment $t$, assuming values of the measured branch currents $i_{b i}(i=1,2, . .9)$ remain constant in a control period $T_{p}$, with the injection of CMV, the predicted equivalent capacitor-voltage changes are written as

$$
\Delta u_{c i, e q}^{c o m}=\frac{v_{b i, r e f, c o m}^{p u} \cdot i_{b i} \cdot T_{p}}{C_{e q}}=\frac{\left(v_{b i, r e f}^{p u}-v_{c o m}^{p u}\right) \cdot i_{b i} \cdot T_{p}}{C_{e q}}, i=1,2, . .9
$$

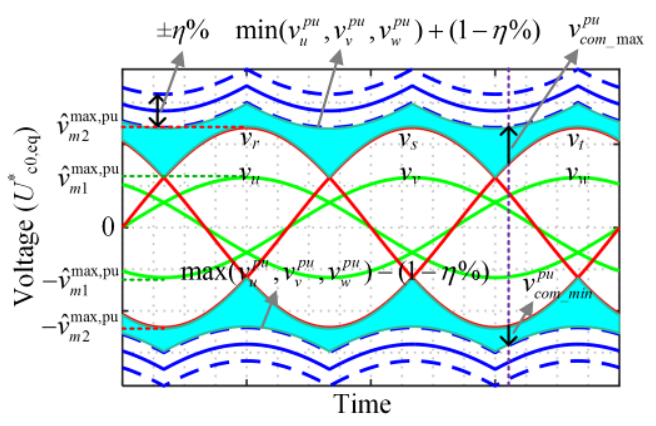

(a)

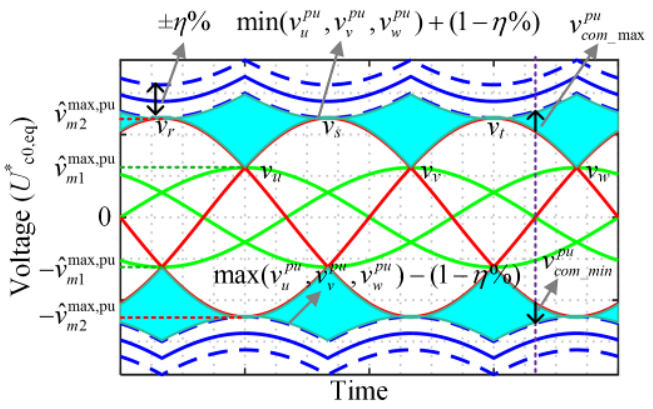

(b)

Fig. 4. The range of the applicable CMV. (a) $\hat{v}_{m 1}=\hat{v}_{m 1}^{\max }, \hat{v}_{m 2}=\hat{v}_{m 2}^{\max }, \xi=1, f_{1}=f_{2}$, $\theta=0$, (b) $\hat{v}_{m 1}=\hat{v}_{m 1}^{\max }, \hat{v}_{m 2}=\hat{v}_{m 2}^{\max }, \xi=1, f_{1}=f_{2}, \theta=180^{\circ}$.

To suppress the capacitor-voltage fluctuation, an objective function is set up in (18) where $U_{C 0, e q}^{*}-u_{c i, e q}$ is the equivalent capacitor-voltage error on branch $i$. Clearly, the capacitor-voltage fluctuation can be realized by the decrease of function $J$ which is the function of CMV and branch currents. In this part, we assume branch currents $i_{b i}(i=1,2, . .9)$ remain constant in a control period and the value of them are the same as the measured value at the beginning of the control period. Therefore, the CMV is the only variable in function $J$ as $J=J\left(v_{c o m}^{p u}\right)$.

$$
\begin{aligned}
& \min J=\sum_{i=1}^{9}[\left(U_{C 0, e q}^{*}-u_{c i, e q}\right)-\underbrace{\left(v_{b i, r e f}^{p u}-v_{c o m}^{p u}\right) \cdot i_{b i} \cdot T_{p} / C_{e q}}_{\Delta u_{c i, e q}^{c o m}}]^{2} \\
& \text { s.t. } v_{c o m}^{p u}=v_{c o m}^{p u, j}, j=0,1, . . N_{c o m} .
\end{aligned}
$$

Among the applicable CMVs in (16), the CMV that minimizes the function $J$ contributes the most to the balancing of capacitor-voltages in the $\mathrm{M} 3 \mathrm{C}$. As a result, the injected CMV can be chosen from $v_{c o m}^{p u, j}\left(j=0,1, . . N_{c o m}\right)$ which minimizes the function $J$. Obviously, the value of $N_{\text {com }}$ influences the effectiveness of the balancing control. A large $N_{\text {com }}$ contributes better to the balancing control but will lead to a longer computation time. Assuming that function $J$ in (18) gets the minimum value when $j=k$, the optimized CMV is then denoted as $v_{c o m}^{p u, k}$. As can be seen in Fig. 4, the proposed CMV optimization method can fully utilize the applicable CMV range at each control moment.

\section{B. Optimal circulating currents injection}

Besides the CMV, the injection of inner circulating currents is the other control degree. In Section III-A, by the optimization of CMV, the function $J$ in (18) is reduced to the value of $J=J\left(v_{c o m}^{p u}=v_{c o m}^{p u, k}\right)$ with the assumption that branch currents $i_{b i}$ $(i=1,2, . .9)$ remain constant as the measured value in a control period. In this part, based on the optimized CMV of $v_{c o m}^{p u, k}$ provided in Section III-A, the design of the inner circulating currents will be discussed to further reduce the value of function $J$.

In the $\mathrm{M} 3 \mathrm{C}$, branch currents can be divided into two parts, the basic branch currents in (4) and the additional circulating currents in (19). In combination, the branch currents are written in (20).

$$
\begin{aligned}
& {\left[\mathbf{i}_{\mathrm{cir}}\right]=\left[\begin{array}{lllllllll}
i_{c i r, 1} & i_{c i r, 2} & i_{c i r, 3} & i_{c i r, 4} & i_{c i r, 5} & i_{c i r, 6} & i_{c i r, 7} & i_{c i r, 8} & i_{c i r, 9}
\end{array}\right]^{T} .} \\
& i_{b i}=i_{b 0, i}+i_{c i r, i}, i=1,2, . .9
\end{aligned}
$$

Combining the injection of the optimized CMV $v_{c o m}^{p u, k}$ and a set of additional circulating currents $\left[\mathbf{i}_{\text {cir }}\right]$, the predicted equivalent capacitor-voltage changes in a control period are written as

$$
\Delta u_{c i, e q}^{c o m, i c i r}=\frac{\left(v_{b i, r e f}^{p u}-v_{c o m}^{p u, k}\right) \cdot\left(i_{c i r, i}+i_{b 0, i}\right) \cdot T_{p}}{C_{e q}}, i=1,2, . .9
$$

Since CMV is fixed as $v_{c o m}^{p u, k}$ here, function $J$ in (18) is now the function of inner circulating currents. Combined with (20) and (21), the function in (18) is rewritten in (22) where additional circulating currents $\left[\mathbf{i}_{\text {cir }}\right]$ are the optimization variables. Clearly, the set of additional circulating currents that minimizes the function $J$ in (22) contributes the most to the balancing of capacitor-voltages in the $\mathrm{M} 3 \mathrm{C}$. 


$$
\begin{aligned}
& \min J=\sum_{i=1}^{9}[\left(U_{c 0, e q}^{*}-u_{c i, e q}\right)-\underbrace{\left.\frac{\left(v_{b i, r e f}^{p u}-v_{c o m}^{p l, k}\right)\left(i_{c i r, i}+i_{b 0, i}\right) T_{p}}{C_{e q}}\right]^{2}}_{\Delta u_{c i, e q}^{c i o c i r}} \\
& \text { s.t. } \mathbf{A}\left[\mathbf{i}_{\text {cir }}\right]=\mathbf{b} \\
& -\xi \cdot I_{c i r, M A X} \leq i_{c i r, i} \leq \xi \cdot I_{c i r, M A X},
\end{aligned}
$$

where

$$
A=\left[\begin{array}{lllllllll}
1 & 1 & 1 & 0 & 0 & 0 & 0 & 0 & 0 \\
0 & 0 & 0 & 1 & 1 & 1 & 0 & 0 & 0 \\
1 & 0 & 0 & 1 & 0 & 0 & 1 & 0 & 0 \\
0 & 1 & 0 & 0 & 1 & 0 & 0 & 1 & 0 \\
1 & 1 & 1 & 1 & 1 & 1 & 1 & 1 & 1
\end{array}\right], b=\left[\begin{array}{l}
0 \\
0 \\
0 \\
0 \\
0
\end{array}\right]
$$

It should be noted that the nine additional circulating currents could not be optimized independently because only four independent circulating currents exist in the $\mathrm{M} 3 \mathrm{C}$ as explained in Section II-A. Therefore, equality constraints are included in (22) to ensure that the circulating current injection will not affect the input and output currents $\left(i_{u}, i_{v}, i_{w}\right.$ and $\left.i_{r}, i_{s}, i_{t}\right)$. In addition, the inequality constraints are also included in (22) to limit branch current stresses. Accordingly, the applicable range of the circulating current on a certain branch is shown in light blue filling in Fig. 5.

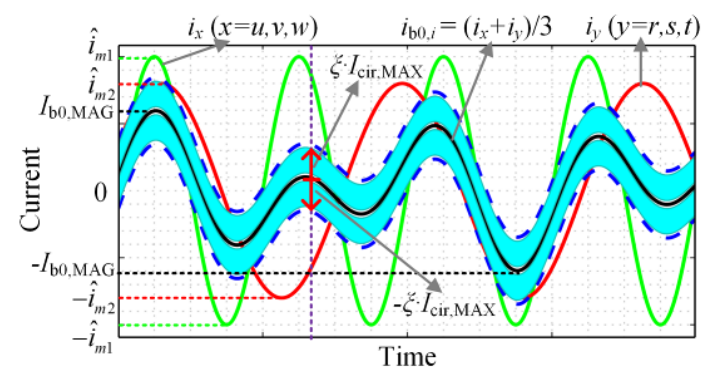

Fig. 5. The range of the applicable circulating current for a certain branch.

In Fig. 5, $I_{\mathrm{b} 0, M A G}$ is the magnitude of the branch current when no circulating currents are used. According to (4), I $I_{\mathrm{b} 0, M A G}$ satisfies

$$
I_{b 0, M A G}=\left(\hat{i}_{m 1}+\hat{i}_{m 2}\right) / 3
$$

Similar to the limitation of CMV in (14) and (15), $\xi(\xi \in[0,1])$ is used as an adjustable factor to limit the injection of circulating currents. Theoretically, the best circulating current injection could be obtained by solving the optimization problem in (22), which is non-linear and with constraints. However, it is difficult to get the global optimum solution due to the complexity of the problem, so a sub-optimized solution is designed and used in this paper. Firstly, neglecting all the equality and inequality constraints in (22), then the optimized additional circulating current references satisfy (25), where combined with the injected CMV of $v_{c o m}^{p u, k}$, the set of circulating currents $i_{c i r, i}^{*}(i=1,2, . .9)$ in (25) can reduce the value of function $J$ to zero (i.e. no capacitor-voltage fluctuation).

$$
J=0 \Rightarrow i_{c i r, i}^{*}=\frac{\left(U_{C 0, e q}^{*}-u_{c i, e q}\right) \cdot C_{e q}}{\left(v_{b i, r e f}^{p u}-v_{c o m}^{p u, k}\right) \cdot T_{p}}-i_{b 0, i}, i=1,2, . .9
$$

However, results in (25) assume $i_{c i r, 1}, i_{c i r, 2}, . . i_{c i r, 9}$ as nine independent currents so a direct application of (25) will influence the input and output currents $\left(i_{u}, i_{v}, i_{w}\right.$ and $\left.i_{r}, i_{s}, i_{t}\right)$. The application of circulating currents in (25) also can seriously increase the branch current stresses. Hereby, in this paper a sub-optimized solution of (22) is designed by applying an additional term to the circulating currents in (25). For each branch, the additional term includes parts of circulating currents from other branches as shown in (26).

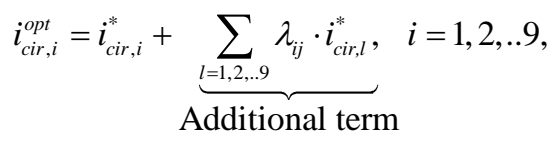

where

$$
\begin{aligned}
& {\left[\begin{array}{llll}
\lambda_{11} & \lambda_{12} & \cdots & \lambda_{19} \\
\vdots & \vdots & \ddots & \vdots \\
\lambda_{91} & \lambda_{92} & \ldots & \lambda_{99}
\end{array}\right]=\left[\begin{array}{lll}
\mathbf{C}_{1} & \mathbf{C}_{2} & \mathbf{C}_{2} \\
\mathbf{C}_{2} & \mathbf{C}_{1} & \mathbf{C}_{2} \\
\mathbf{C}_{2} & \mathbf{C}_{2} & \mathbf{C}_{1}
\end{array}\right],} \\
& \mathbf{C}_{1}=-\left[\begin{array}{ccc}
0 & 0.5 & 0.5 \\
0.5 & 0 & 0.5 \\
0.5 & 0.5 & 0
\end{array}\right], \mathbf{C}_{2}=\left[\begin{array}{ccc}
-0.5 & 0.25 & 0.25 \\
0.25 & -0.5 & 0.25 \\
0.25 & 0.25 & -0.5
\end{array}\right] .
\end{aligned}
$$

It can be proved that with the additional term, the set of circulating currents $\left[\mathbf{i}_{\text {cir }}^{\text {opt }}\right]$ in (26) satisfy the equality constraints in (22). Meanwhile, the inequality constraints in (22) can be realized by limiting the value of injected circulating currents in (26).

\section{Synthesized control scheme}

The realization of the proposed control consists of two steps as shown in Fig. 6 (a). The first step is to determine the optimized injection of CMV $v_{c o m}^{p u, k}$ and the second step is to calculate the optimal circulating currents. In Fig. 6 (a), in the first step, first calculate the range of CMV. Then discretize the range and search the optimized CMV $v_{c o m}^{p u, k}$ to minimize function $J$ in (18) where CMV is the only variable in $J$ because the branch currents in (18) are considered constant in the first step. In Fig. 6 (a), in the second step, as the CMV has been chosen as $v_{c o m}^{p u}=v_{c o m}^{p u, k}$, circulating currents are now becoming the only variable in $J$. Then first calculate the additional circulating currents $i^{*}{ }_{c i r, i}(i=1,2, . .9)$ in (25). On the basis of the calculated $i_{c i r, i}^{*}(i=1,2, . .9)$, in order to satisfy the equality constraints in (22), a sub-optimized solution is calculated according to (26) where a set of additional terms is included. Additionally, to satisfy the inequality constrains in (22), circulating current references are limited in the range of $\left[-\xi \cdot I_{\mathrm{cir}, \mathrm{MAX}}, \xi \cdot I_{\mathrm{cir}, \mathrm{MAX}}\right]$ to limit branch current stresses. 
The proposed control realizes the capacitor-voltage balancing by continuously decreasing the value of the objective function $J$ as shown in Fig. 6 (b). In the proposed strategy, the decrease of function $J$ is shown in red bolded arrow in Fig. 6 (b). In the first step, by the injection of CMV, the value of $J$ decreases from $J\left(v_{c o m}^{p u}=0\right)$ to $J\left(v_{c o m}^{p u}=v_{c o m}^{p u, k}\right)$. In the second step, by the injection of circulating currents the value of $J$ decreases from $J\left(v_{c o m}^{p u}=v_{c o m}^{p u, k}\right)$ to $J\left(v_{c o m}^{p u}=v_{c o m}^{p u, k},\left[\mathbf{i}_{\text {cir }}\right]=\left[\mathbf{i}_{\text {cir }}^{\text {opt }}\right]\right)$. The green arrow in step 2 shows the injection effect of circulating currents in (25) which minimizes the value of function $J$ to be zero $(J=0)$. However, as aforementioned, this injection can increase branch current stresses and influence the input and output system currents. The sub-optimized circulating currents avoid these negative effects but the price is an increase of $\Delta J$ shown in purple in Fig. 6 (b). Moreover, it should be noted that in some extreme conditions, there is possibility that the application of additional term in (26) increases $J$ instead of decreasing it as shown in Fig. 6 (b) with brown arrow and a red cross, which indicates the worsening of capacitor-voltage fluctuation. So in practice, after the calculation of circulating currents in (26), the value of function $J\left(v_{c o m}^{p u}=v_{c o m}^{p u, k},\left[\mathbf{i}_{\text {cir }}\right]=\left[\mathbf{i}_{\text {cir }}^{\text {opt }}\right]\right)$ need to be calculated and assessed. If the value is larger than $J\left(v_{c o m}^{p u}=v_{c o m}^{p u, k}\right)$, the second step of circulating current injection will be skipped.

It is worth mentioning that in the proposed control, the optimization of CMV and circulating currents are realized separately and in sequence. It is possible to optimize these two control degrees together but it will lead to much higher control complexity and hence the control becomes difficult to realize in practice.

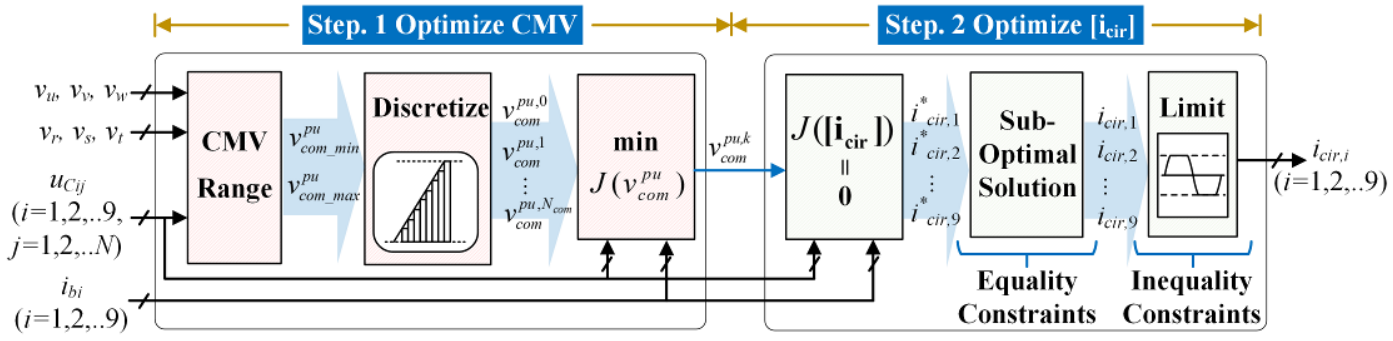

(a)

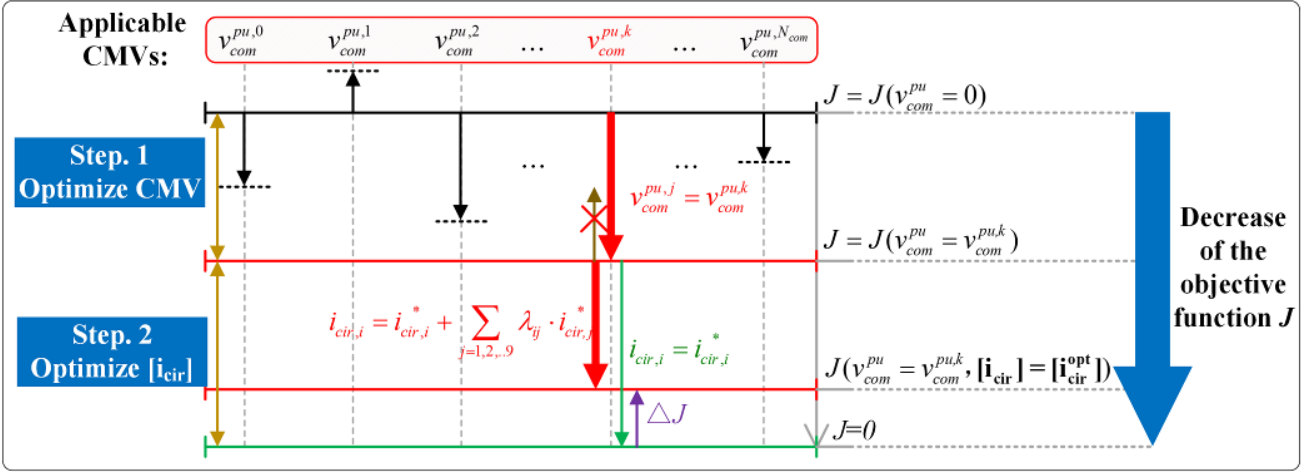

(b)

Fig. 6. The optimization process in the proposed control. (a) Control flow, (b) Decrease of the objective function $J$.

\section{The restriction of $C M V$ and circulating currents}

The injection of CMV and circulating currents may cause problems like motor bearing failure, increased branch current stresses and lower system efficiency. This section proposes a CMV and circulating current limitation method to reduce the negative effects caused by the injection. In the $\mathrm{M} 3 \mathrm{C}$, with the injection of CMV and circulating currents, the nine branch powers are written in (28). Branch power $p_{b i}$ consists of two components, the basic branch power $p_{b 0, i}$ in (7) and the adjusted branch power $p_{b \_a d j, i}$.

$$
\begin{aligned}
& p_{b i}=v_{b i} i_{b i}=\left(v_{x}-v_{y}-v_{c o m}\right) \cdot\left(i_{b 0, i}+i_{c i r, i}\right)= \\
& \underbrace{\left(v_{x}-v_{y}\right) i_{b 0, i}}_{p_{b 0, i}}+\underbrace{\left(v_{x}-v_{y}\right) i_{c i r, i}-v_{c o m} i_{b 0, i}-v_{c o m} i_{c i r, i}}_{p_{b_{-} a d j, i}}, i=1,2, . .9 .
\end{aligned}
$$

The injection of CMV and circulating currents, as explained in Section III-A, B and C, actually helps realizing the capacitor-voltage balancing by introducing appropriate adjusted branch powers in (28). When the M3C is operated away from critical-frequency points, theoretically the capacitor voltages can be balanced without additional control (without injection of CMV or circulating currents). However, due to factors like modulation or small calculation inaccuracy, slight power differences exist among nine branches. In this case, only limited injection of CMV and circulating currents are necessary for creating small adjusted branch power to eliminate the slight branch power differences. As a result, the magnitude of the CMV and circulating currents should be limited which is realized by reducing the value of factor $\xi$ in (14), (15) and (22) to $\xi_{0}\left(0 \leq \xi_{0}<1\right)$.

When the $\mathrm{M} 3 \mathrm{C}$ is operated at or around critical-frequency points, a dc or very low-frequency power components $\Delta p_{b i}^{d c}$ is introduced as explained in Section II-B. The proposed control 
should eliminate these power components. Taking the condition of ' $f_{1} \approx f_{2}$ ' as an example, according to (8), branch power component $p_{f 1-f 2}$ will cause large branch energy fluctuation. Assuming that the adjusted branch power $p_{b_{-} a d j}$ needs to restore branch energy from the largest energy deviation to its set value in time period of $\Delta t$, combined with (8), $p_{b \_a d j}$ need to satisfy (29).

$$
p_{b_{-} a d j} \Delta t=\frac{1}{2} \Delta E_{p-p} \approx \frac{1}{2}\left|\frac{\hat{p}_{f_{1}-f_{2}}}{\pi f_{1}-\pi f_{2}}\right|
$$

In (28), since the injected CMV and circulating currents are comparatively small compared to voltage differences $v_{x}-v_{y}$ and basic branch currents $i_{b 0, i}$, the product of $v_{c o m}$ and $i_{c i r, i}$ can be neglected. Hereby factor $\xi$ which decides the range of the injected CMV and circulating current is set to be inversely proportional to the frequency of $f_{1}-f_{2}$ as in (30).

$$
\left.\begin{array}{l}
p_{b_{-} a d j} \Delta t \approx \frac{1}{2}\left|\frac{\hat{p}_{f_{1}-f_{2}}}{\pi f_{1}-\pi f_{2}}\right| \\
p_{b_{-} a d j, i} \approx\left(v_{x}-v_{y}\right) i_{c i r, i}-v_{c o m} i_{b 0, i}
\end{array}\right\} \Rightarrow \xi \propto \frac{1}{\left|f_{1}-f_{2}\right|}
$$

Considering the whole frequency range, factor $\xi$ is finally configured as (31) and the relationship between output frequency and factor $\xi$ is showed in Fig. 7. The value of $\Delta f^{*}$ determines the width of the frequency range around critical-frequency points where the maximum CMV and circulating-current should be injected. The input side is assumed to be connected with the $\mathrm{AC}$ gird, so $f_{1}$ is fixed in (31).

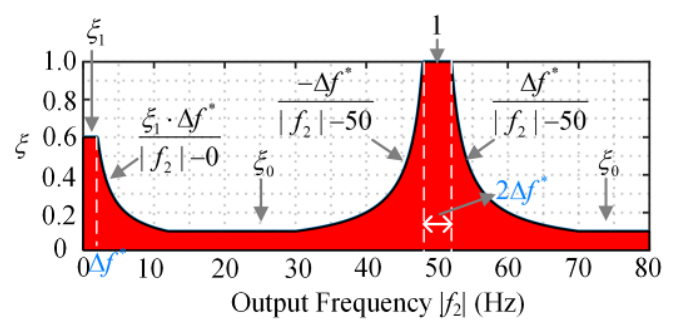

Fig. 7. Limitation of the CMV and circulating-currents $\left(f_{1}=50 \mathrm{~Hz}\right)$.

$$
\begin{aligned}
& \xi= \\
& \begin{cases}\xi_{1} & \left|f_{2}\right| \leq \Delta f^{*} \\
\xi_{1} \Delta f^{*} /\left(\left|f_{2}\right|-0\right) & \Delta f^{*} \leq\left|f_{2}\right| \leq\left(\xi_{1} / \xi_{0}\right) \Delta f^{*} \\
\xi_{0} & \left(\xi_{1} / \xi_{0}\right) \Delta f^{*} \leq\left|f_{2}\right| \leq f_{1}-\left(1 / \xi_{0}\right) \Delta f^{*} \\
-\Delta f^{*} /\left(\left|f_{2}\right|-f_{1}\right) & f_{1}-\left(1 / \xi_{0}\right) \Delta f^{*} \leq\left|f_{2}\right| \leq f_{1}-\Delta f^{*} \\
1 & f_{1}-\Delta f^{*} \leq\left|f_{2}\right| \leq f_{1}+\Delta f^{*} \\
\Delta f^{*} /\left(\left|f_{2}\right|-f_{1}\right) & f_{1}+\Delta f^{*} \leq\left|f_{2}\right| \leq f_{1}+\left(1 / \xi_{0}\right) \Delta f^{*} \\
\xi_{0} & \left|f_{2}\right| \geq f_{1}+\left(1 / \xi_{0}\right) \Delta f^{*}\end{cases}
\end{aligned}
$$

When $f_{2}= \pm f_{1}$, the factor $\xi$ is set to be 1 to create a maximum adjusted power. When $f_{2}=0$, the factor $\xi$ is configured as $\xi_{1}$ $\left(0 \leq \xi_{1}<1\right)$. The value of $\xi_{1}$ depends on the required load power. For instance, if the load is an electrical motor operated with constant-torque, $\xi_{1}$ should be configured smaller than ' 1 ', since the motor power is relatively small at low-speed. When M3C is operated away from critical-frequency points, the factor $\xi$ is reduced to a small value of $\xi_{0}$ as aforementioned.

\section{OVERALl CONTROL STRATEGY}

In the $\mathrm{M} 3 \mathrm{C}$, the capacitor-voltage balancing control consists of three levels:

1) Overall-balancing control regulates the average value of all cell capacitor-voltages to the voltage command by maintaining the dc branch power $p_{d c}$ in (7) to be zero;

2) Branch-balancing control balances the average value of capacitor-voltages among the nine branches. In this paper, an optimization based predictive control method is proposed to realize the branch-balancing control;

3) Individual-balancing control balances cell capacitor-voltages inside each branch. As explained at the beginning of Section III, many well-constructed methods could be employed for this control.

The overall control block is shown in Fig. 8. The input side of the $\mathrm{M} 3 \mathrm{C}$ is assumed to be connected with the $\mathrm{AC}$ grid. The overall-balancing control includes the grid-side control and the load-side control. The load-side control provides the output voltage references $v_{r}, v_{s}, v_{t}$ according to the load requirements (motor speed, torque and etc.). The grid-side control realizes the active power balancing between the input and output system and ensure unity power factor at AC grid. The grid-side control provides the input voltage references $v_{u}, v_{v}, v_{w}$. In this paper, a traditional d-q frame based vector-control [29] is applied as the grid-side control.

The branch-balancing control regulates the average capacitor voltages among the nine branches. In Fig. 8, the proposed optimization based predictive control method is used as explained in Section III. The proposed control provides the branch output voltage references $v_{b i, r e f, c o m}^{p u, k}(i=1,2, . .9)$ and branch circulating current references $i_{c i r, i}(i=1,2, . .9)$. The 'inner circulating current control' regulates actual circulating currents to follow the references $i_{c i r, i}(i=1,2, . .9)$. The detailed circulating current control block is shown in Fig. 9. This regulation is performed in double $\alpha \beta$ frame by adjusting the voltage of $v_{\alpha \alpha}$, $v_{\alpha \beta}, v_{\beta \alpha}$ and $v_{\beta \beta}$ in $\left[\mathbf{v}_{\mathbf{b}}\right]_{\mathbf{D}-\alpha \beta}$ in (3) by four proportional regulators according to the circulating current errors, the same method as depicted in [8]. A set of branch voltage adjustments (denoted as $\Delta v_{c i r, i}^{p u}$ ) used for circulating current control is added to the branch output voltage references. The final branch output voltage references $v_{b i}^{*}(i=1,2, . .9)$ are the sum of $v_{b i, r e f, c o m}^{p u, k}$ and $\Delta v_{c i r i}^{p u}$. The individual-balancing control is then performed based on these voltage references.

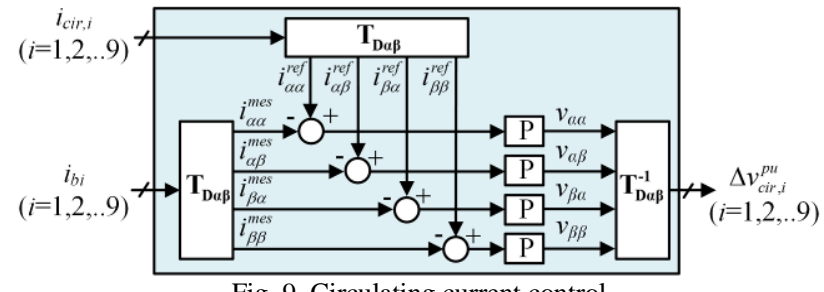

Fig. 9. Circulating current control 
The Individual-balancing control balances cell capacitor-voltages in each branch. In this paper, the Individual-balancing control utilizes PD-PWM method and

uses cell capacitor-voltage sorting as the balancing strategy, as the same method in [8].

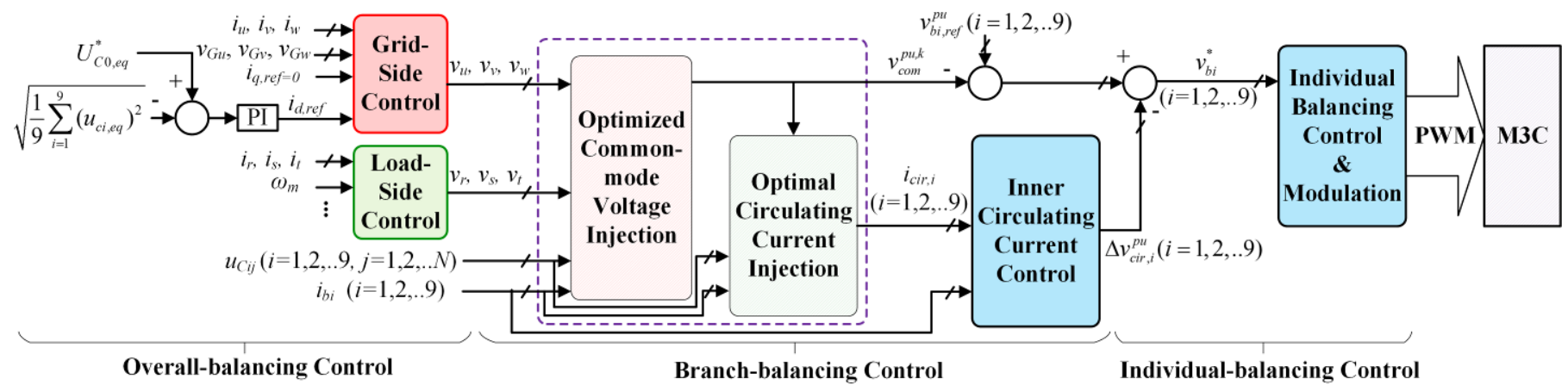

Fig. 8. Overall control block of the proposed control strategy.

\section{EXPERIMENT RESULTS}

\section{A. System configuration}

A downscaled M3C prototype with $N=3$ full-bridge cells per branch was build up to validate the proposed control strategy. The configuration of the control system and the experiment platform are shown in Fig. 10.

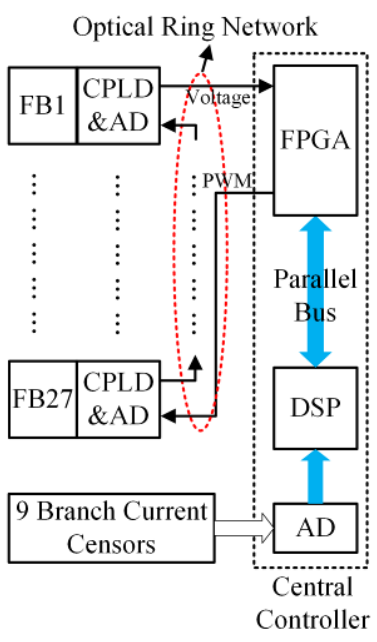

(a)

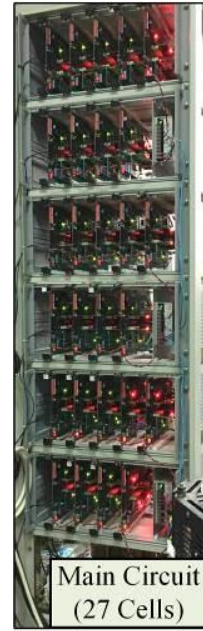

(b)

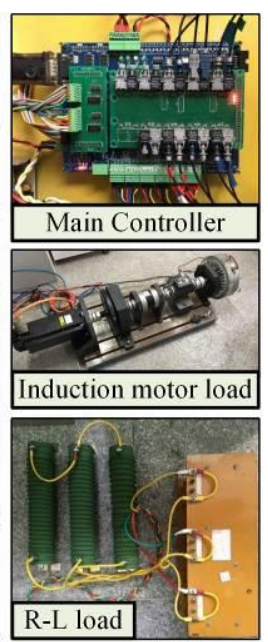

(b)
Fig. 10. The M3C Experiment Platform. (a) Control system structure, (b) Photos of the M3C prototype, clockwise from left: Main circuit, Main controller, Induction motor load, R-L load.

The M3C prototype consists of a central controller and 27 full-bridge cells. The central controller includes a 32-bit floating-point digital signal processor TMS320F28377 and a field-programmable gate array (FPGA) chip. Each full-bridge has a complex programmable logic device (CPLD) based cell controller. As shown in Fig. 10 (a), central controller and cell controllers are connected in an optical-fiber-based ring network. PWM signals and capacitor voltage feedbacks are transmitted through this network. Table II summarizes the circuit parameters used for the experiment. The input (AC grid) voltage magnitude $\hat{v}_{m 1}$ is constant at $160 \mathrm{~V}$ in the experiments. Considering the maximum output voltage magnitude $\hat{v}_{m 2}^{\max }$ as $250 \mathrm{~V}$ and the maximum capacitor-voltage fluctuation $\pm \eta \%$ as $\pm 10 \%$, the reference voltage of each module capacitor is set as $\mathrm{U}_{\mathrm{C}}{ }^{*}=155 \mathrm{~V}$ according to (10). The maximum value of the circulating current $I_{c i r, M A X}$ in (22) is set as 2 A.

TABLE II

Experiment Parameters

\begin{tabular}{|c|c|c|}
\hline Parameters & Symbols & Value \\
\hline Switching Frequency & $f_{\mathrm{s}}$ & $2 \mathrm{kHz}$ \\
\hline Full-bridge cells per branch & $N$ & 3 \\
\hline Module Capacitance & $C$ & $880 \mathrm{uF}$ \\
\hline Branch inductance & $L_{b}$ & $2 \mathrm{mH}$ \\
\hline AC grid inductance & $L_{s}$ & $5 \mathrm{mH}$ \\
\hline Capacitor Voltage & $U_{C} *$ & $155 \mathrm{~V}$ \\
\hline Input frequency & $f_{1}$ & $50 \mathrm{~Hz}$ \\
\hline Input Voltage Magnitude & $\hat{v}_{m 1}$ & $160 \mathrm{~V}$ \\
\hline Load Resistance & $R$ & $37 \Omega$ \\
\hline Load Reluctance & $L$ & $10 \mathrm{mH}$ \\
\hline
\end{tabular}

\section{B. Control strategy verification with $R$-L load}

Fig. 11 to Fig. 14 show the experimental results with the R-L load, the output voltage magnitude $\hat{v}_{m 2}$ is set as $250 \mathrm{~V}$. The limitation coefficient in (31) are set as ' $\xi_{1}=1, \xi_{0}=0.15$, $\Delta f^{*}=2 \mathrm{~Hz}$ '. Parameter $\xi_{1}$ is set as ' 1 ' because large load power is required when the output frequency is zero. In Fig. 11, the output frequency $f_{2}$ is 0 . As shown in Fig. 11 (d), before time $t_{1}$, the proposed control is applied. Capacitor-voltages coincide to each other and fluctuate around $155 \mathrm{~V}$. At time $t_{1}$, the injection of circulating currents and CMV is removed. The capacitor-voltages become unbalanced quickly which consists with the analysis and result in Fig. 2 (a). At time $t_{2}$, the proposed control is restored and the capacitor-voltages become balanced quickly. Fig. 11 (b) and Fig. 11 (c) present AC grid voltages $v_{G u}, v_{G v}, v_{G w}$ (voltage definition is shown in Fig. 1 (a)) and input currents $i_{u}, i_{v}, i_{w}$. As input currents are in phase with AC grid voltages, no reactive power is applied at the AC grid. Fig. 11 (e) shows the waveforms of branch currents. It can be seen that there are some high-frequency harmonics on branch currents, which are introduced by the circulating current injection. Due to the using of sub-optimized circulating current solution in (26), the harmonics on branch currents will not appear on the input and output currents. Fig. 11 (f) shows the waveform of the injected CMV. In time period $t_{1} \leq t \leq t_{2}$, as the CMV injection is removed, only very small CMV exists which 
is caused by modulation. Before time $t_{1}$ and after time $t_{2}$, a CMV around $\pm 250 \mathrm{~V}$ is injected to realize the branch-balancing control. For this particular operating point, a comparison of branch current magnitudes with existing control methods is presented in Table. III. As can be seen, the proposed control can significantly reduce the branch current stress when the output-frequency is zero.

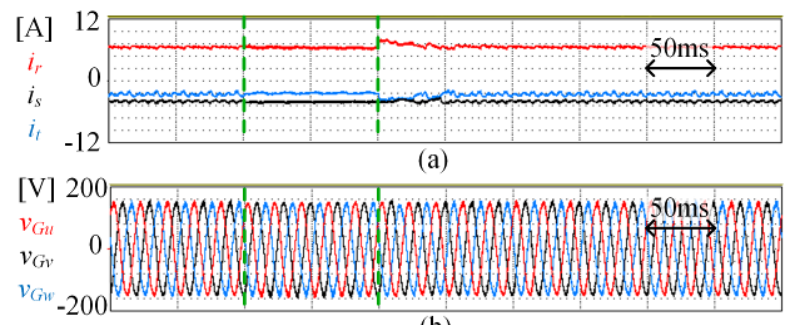

(b)
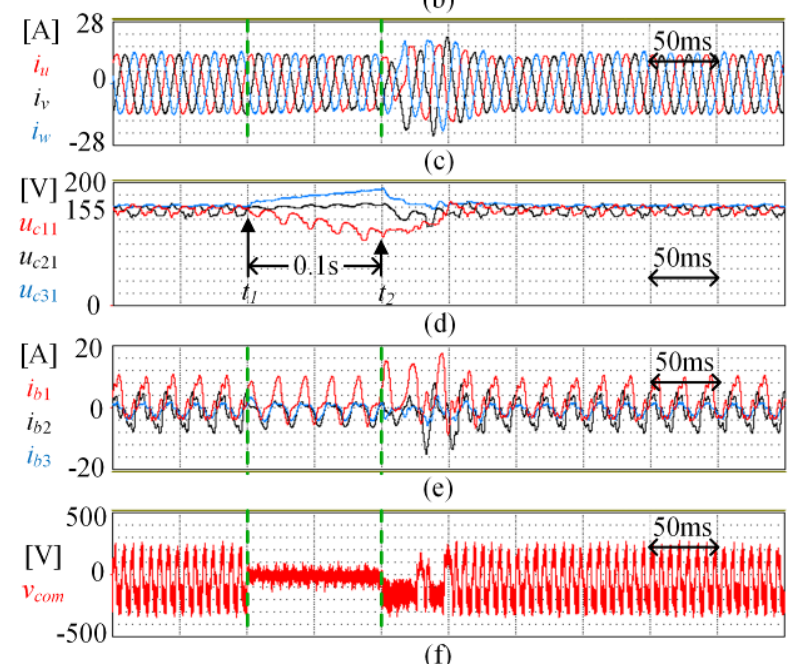

(f)

Fig. 11. Capacitor voltages balancing verification with R-L load $\left(f_{1}=50 \mathrm{~Hz}\right.$, $f_{2}=0 \mathrm{~Hz}$ ). (a) Output currents. (b) AC grid phase-to-neutral voltages. (c) Input currents. (d) Capacitor voltages. (e) Branch currents. (f) The CMV.

TABLE III

Branch current amplitude comparison $\left(f_{1}=50 \mathrm{~Hz}, f_{2}=0 \mathrm{~Hz}\right)$

\begin{tabular}{cccc}
\hline \hline $\begin{array}{c}\text { Basic branch current } \\
\left(i_{x}+i_{y}\right) / 3\end{array}$ & Method in [22] & Method in [8] & Proposed method \\
\hline $6.3 \mathrm{~A}(100 \%)$ & $190.3 \%$ & $229.4 \%$ & $126.9 \%$ \\
\hline
\end{tabular}

A similar verification is also applied for the condition of $f_{2}=50 \mathrm{~Hz}$. In Fig. 12, the proposed control is removed at time $t_{1}$ and restored at time $t_{2}$. Results in Fig. 12 verify the effectiveness of the proposed balancing control. Fig. 12 (b) and (c) prove that there is no reactive power at the AC grid. The high-frequency current harmonics in Fig. 12 (e) are caused by the circulating current injection. Fig. 12 (f) shows the injected CMV. For this particular operating point, a comparison of branch current magnitudes with existing control methods is presented in Table. IV. As can be seen, the proposed control help reducing the branch current stress when the output-frequency is the same as the input-frequency. It is worth noting that in Table. IV, results from the existing method are theoretically calculated and the influence of the current ripple on branch current magnitude has not been included, so in real application the branch current magnitudes of existing control method could be larger.
Results in Fig. 11 and Fig. 12 prove a successful and stable operation of the $\mathrm{M} 3 \mathrm{C}$ for the two most critical-frequency points $\left(f_{2}=0 \mathrm{~Hz}\right.$ and $\left.f_{1}=f_{2}=50 \mathrm{~Hz}\right)$. When $\mathrm{M} 3 \mathrm{C}$ is operated away from these critical-frequency points, the balancing control is still necessary as explained in Section III-D. In Fig. 13, the output frequency is $25 \mathrm{~Hz}$. The proposed control is removed from time $t_{1}$. As shown in Fig. 13 (d1), capacitor-voltages will gradually diverge from the reference value. Compared with the results in Fig. 11 (d) and Fig. 12 (d), capacitor-voltages diverge with a much lower speed. At time $t_{2}$, the proposed control is restored and capacitor-voltages become balanced quickly. Fig. 13 (a2) to Fig. 13 (f2) are the zoom-in results of Fig. 13 (a1) and Fig. 13 (f1) in steady state. It can be seen that the capacitor-voltages are balanced very well and there is no reactive power at the $\mathrm{AC}$ grid.
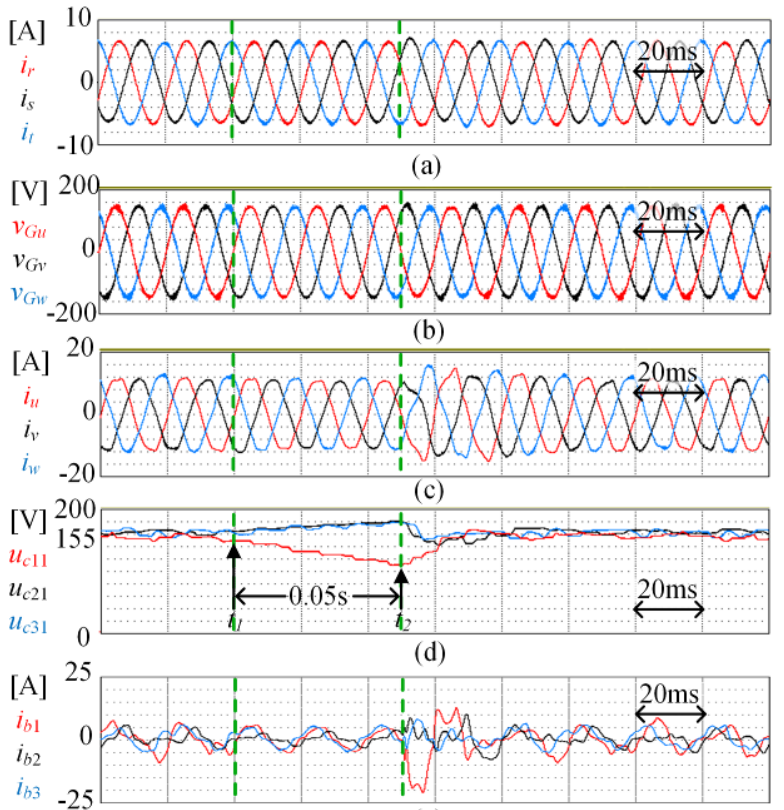

(e)

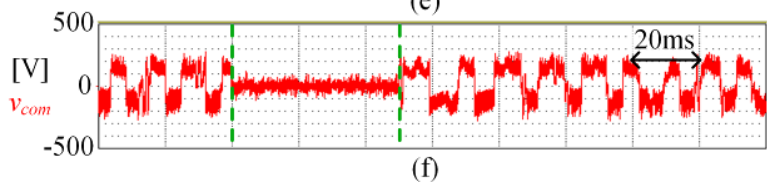

Fig. 12. Capacitor voltages balancing verification with R-L load $\left(f_{1}=50 \mathrm{~Hz}\right.$, $f_{2}=50 \mathrm{~Hz}$ ). (a) Output currents. (b) AC grid phase-to-neutral voltages. (c) Input currents. (d) Capacitor voltages. (e) Branch currents. (f) The CMV.

TABLE IV

Branch current amplitude comparison $\left(f_{1}=50 \mathrm{~Hz}, f_{2}=50 \mathrm{~Hz}\right)$

\begin{tabular}{cccc}
$\begin{array}{c}\text { Basic branch current } \\
\left(i_{x}+i_{y}\right) / 3\end{array}$ & Method in [23] & Method in [30] & Proposed method \\
\hline $6.2 \mathrm{~A}(100 \%)$ & $147.1 \%$ & $158.6 \%$ & $132.2 \%$ \\
\hline
\end{tabular}

Compared with the results in Fig. 11 (e), Fig. 11 (f), Fig. 12 (e) and Fig. 11 (f), the high-frequency branch current harmonics in Fig. 13 (e2) and the CMV in Fig. 13 (f2) are with a much smaller value, because the injection of the circulating currents and CMV is limited according to (31). Fig. 14 (a) and (b) present the output and input line-to-line voltages $v_{\mathrm{rs}}$ and $v_{\mathrm{uv}}$ when output frequency is $25 \mathrm{~Hz}$. Fig. 14 (c) shows the output voltage of the cascaded full-bridges on branch 1 . Since each branch consists of three full-bridges, there are seven voltage levels in Fig. 14 (c). 


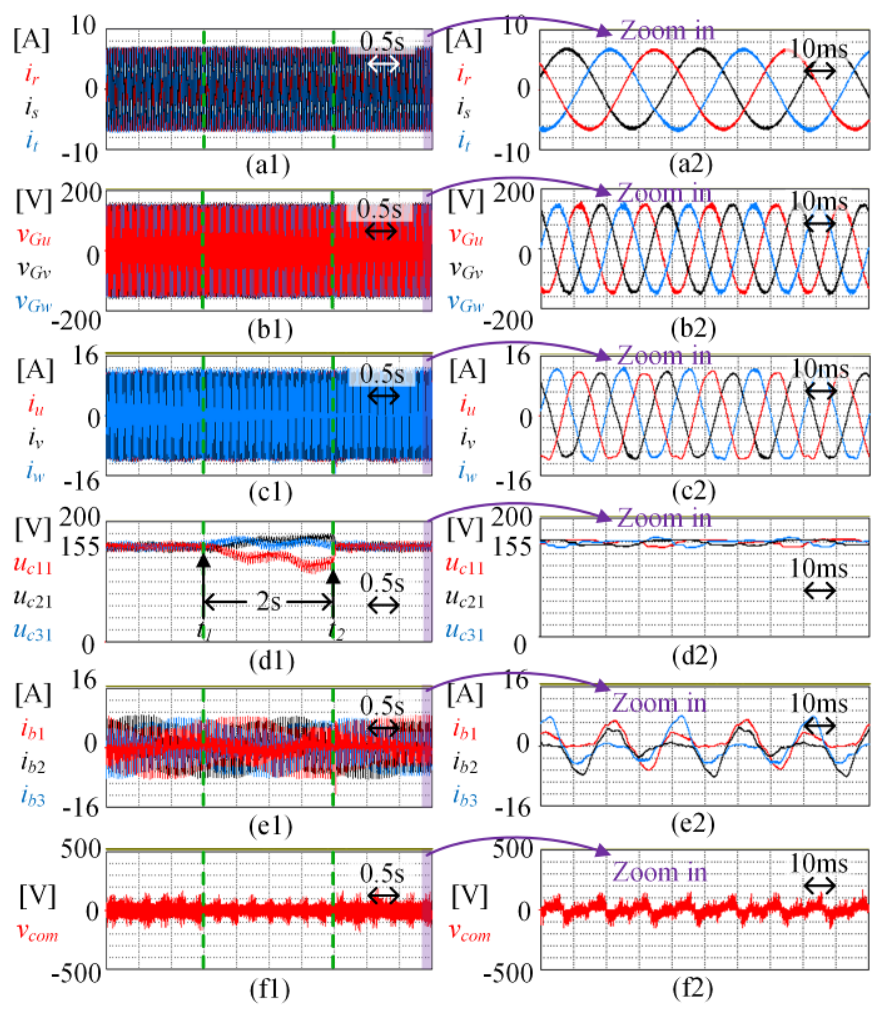

Fig. 13. Capacitor voltages balancing verification with R-L load $\left(f_{1}=50 \mathrm{~Hz}\right.$, $f_{2}=25 \mathrm{~Hz}$ ). (a1) Output currents. (b1) AC grid phase-to-neutral voltages. (c1) Input currents. (d1) Capacitor voltages. (e1) Branch currents. (f1) The CMV. (a2) Output currents. (b2) AC grid phase-to-neutral voltages. (c2) Input currents. (d2) Capacitor voltages. (e2) Branch currents. (f2) The CMV.

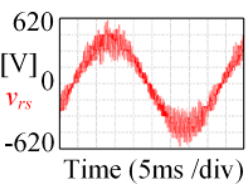

(a)

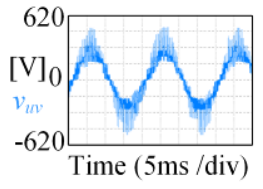

(b)

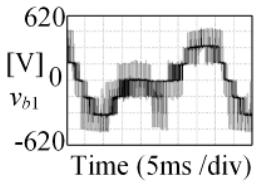

(c)
Fig. 14. Experimental results with R-L load $\left(f_{1}=50 \mathrm{~Hz}, f_{2}=25 \mathrm{~Hz}\right)$. (a) Output-side line-to-line voltage $v_{r s}$. (b) Input-side line-to-line voltage $v_{u v}$. (c) Output voltage of the cascaded full-bridges on branch 1 .

Fig. 15 shows the comparison of the theoretically calculated capacitor-voltage fluctuation without voltage-balancing control and the measured capacitor-voltage fluctuation with proposed control. In Fig. 15, the theoretical capacitor-voltage fluctuation, shown in blue, is calculated according to (8). The measured capacitor-voltage fluctuation is shown in red triangles. In Fig. 15, the measured capacitor-voltage fluctuation magnitude remains a similar value at different output frequency due to the application of the proposed control method. Around critical-frequency points $\left(f_{2}=0 \mathrm{~Hz}\right.$ or $\left.50 \mathrm{~Hz}\right)$, theoretically large capacitor-voltage fluctuation is effectively suppressed with the proposed control method. When the output frequency is away from critical-frequencies, as explained in Section III-D, the injected CMV and circulating currents are limited according to (31) to compensate only small branch power differences caused by modulation or calculation inaccuracy. In this condition, the circulating current and CMV injection will not influence the capacitor-voltage fluctuation. As shown in Fig. 15, when the output frequency is in the range of 10 to $40 \mathrm{~Hz}$ (away from critical-frequency points), the measured capacitor-voltage fluctuation coincides with the theoretical calculated capacitor-voltage fluctuation as predicted.

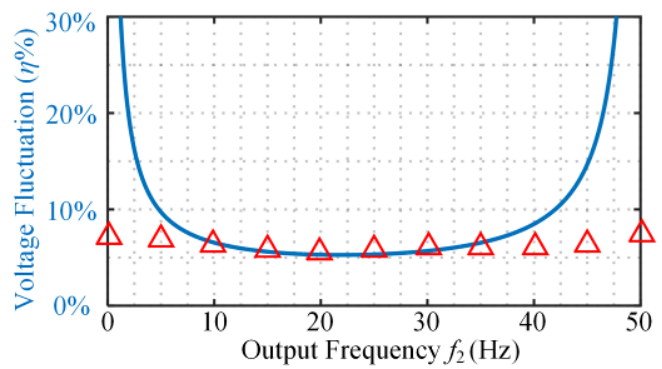

Fig. 15. Verification of the capacitor voltage fluctuation (R-L load,

$$
\hat{v}_{m 2}=250 \mathrm{~V} \text { ). }
$$

\section{Control strategy verification with induction motor load}

The motor-load experiment verifications are performed with a 4 -pole $2.2 \mathrm{~kW}$ induction motor. Specifications of the motor are shown in Table. V. The so-called 'indirect rotor flux orientation' (IRFO) based vector-control is used, which allows an arbitrary instantaneous torque control. The motor is loaded with a constant $60 \%$ rated torque. The limitation coefficient in (31) are set as ' $\boldsymbol{\xi}_{\mathbf{1}}=\mathbf{0 . 3}, \xi_{0}=0.15, \Delta f^{*}=2 \mathrm{~Hz}$ '. Note that since the motor load requires a smaller power at low-speed operation, the value of factor $\xi_{1}$ is reduced to 0.3 .

TABLE V

Parameters of the Induction Motor

\begin{tabular}{ll}
\hline \multicolumn{1}{c}{ Parameters } & \multicolumn{1}{c}{ Value } \\
\hline Rated output power & $2.2 \mathrm{~kW}$ \\
Rated frequency & $50 \mathrm{~Hz}$ \\
Rated rotating speed & $1500 \mathrm{r} / \mathrm{min}$ \\
Rated line-to-line rms voltage & $380 \mathrm{~V}$ \\
Rated stator rms current & $5.3 \mathrm{~A}$ \\
\hline \hline
\end{tabular}

Fig. 16 shows the steady-state experimental waveforms when the motor speed is controlled at $1500 \mathrm{r} / \mathrm{min}$ so the output frequency $f_{2}$ is near the AC Grid frequency $f_{1}\left(f_{2} \approx f_{1}=50 \mathrm{~Hz}\right)$. Fig. 16 (d) proves that with the proposed control method, the capacitor-voltages are well stabilized and balanced at this critical-frequency point.

Fig. 17 shows the experimental start-up performance when the induction motor is loaded with a constant $60 \%$ rated torque. To verify the stable operation of the proposed control at any frequency point, in Fig. 17 the motor is gradually accelerated from 0 to $1500 \mathrm{r} / \mathrm{min}$ in 10 seconds. As shown in Fig. 17 (a), $n^{*}{ }_{r m}$ and $n_{r m}$ are the reference and the measured motor speed. To avoid high start-up currents, a pre-excitation process is applied before acceleration. In the pre-excitation process, the frequency of the output currents is zero as shown in Fig. 17 (b). In the start-up process in Fig. 17, the M3C passes through the two critical operation points $f_{2}=0$ and $f_{2}=50 \mathrm{~Hz}$. Capacitor-voltages shown in Fig. 17 (d) prove the effectiveness of the proposed balancing control method. The magnitude of the injected CMV in Fig. 17 (f) coincides with the limitation in Fig. 7. As the maximum value of the circulating current $I_{c i r, M A X}$ in (22) is set as 2A, compared with the results in [24] there is no obvious increase on branch current magnitude when the motor passing through critical-frequency points in Fig. 17 (e). 


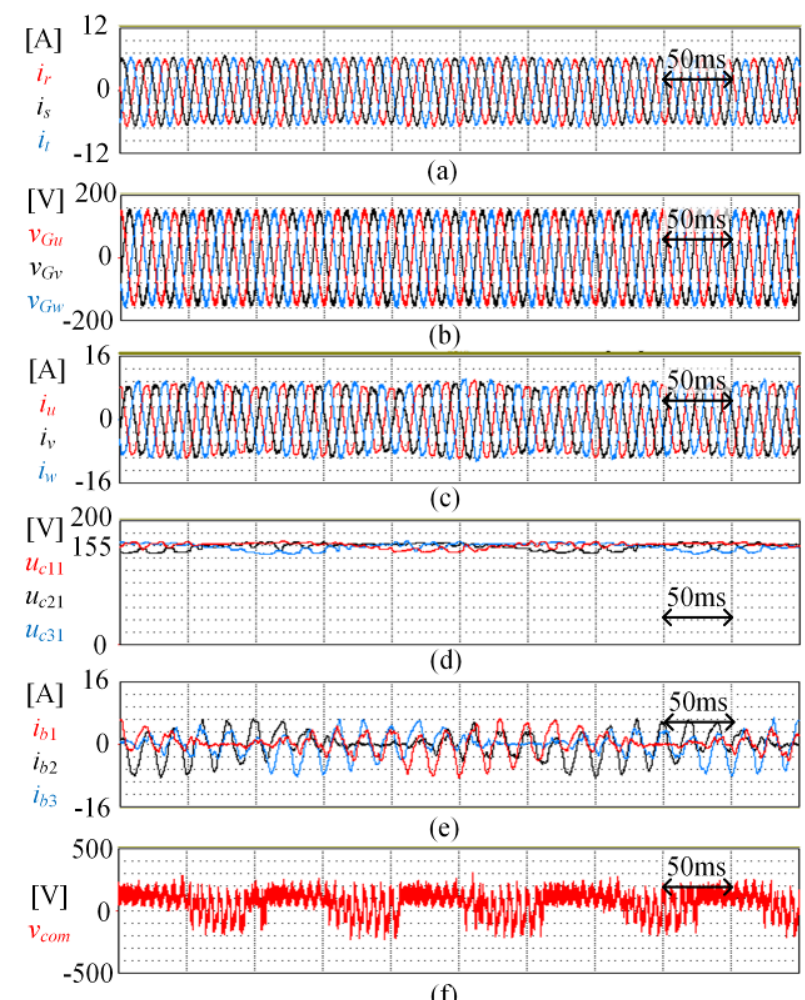

Fig. 16. Experimental results with induction motor load (60\% rated torque, $1500 \mathrm{r} / \mathrm{min}, f_{2} \approx f_{1}=50 \mathrm{~Hz}$ ). (a) Output currents. (b) AC grid phase-to-neutral voltages. (c) Input currents. (d) Capacitor voltages. (e) Branch currents. (f) The CMV.

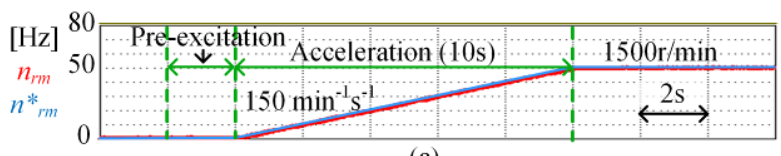

(a)

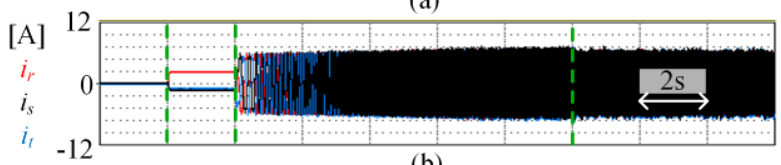

(b)

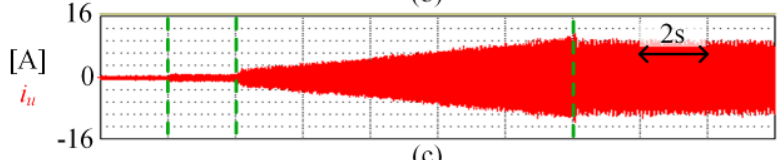

(c)

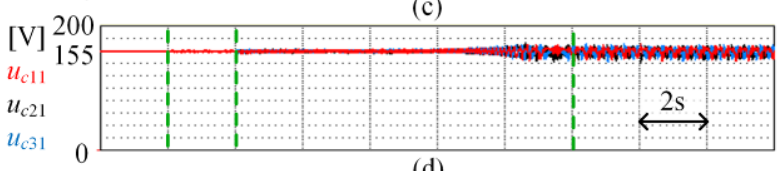

(d)

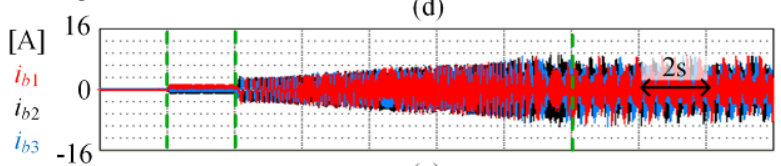

(e)

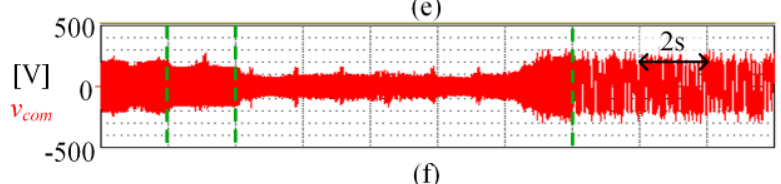

(f)

Fig. 17. The start-up performance ( 0 to $1500 \mathrm{r} / \mathrm{min}$, constant $60 \%$ rated torque).

(a) Motor speed. (b) Output currents. (c) The input current on phase-u. (d) Capacitor voltages. (e) Branch currents. (f) The CMV.

Fig. 18 shows the experimental performance of the motor deceleration and acceleration. In the experiment, the induction motor is loaded with a constant $60 \%$ rated torque. To verify the stable operation of the proposed control with both positive-sequence and negative-sequence load, in Fig. 18 the motor speed is firstly decelerated from $1500 \mathrm{r} / \mathrm{min}$ to $0 \mathrm{r} / \mathrm{min}$ (the motor load is positive-sequence) and then is accelerated to $-1500 \mathrm{r} / \mathrm{min}$ (the motor load is negative-sequence). As shown in Fig. 18 (a), $n_{r m}^{*}$ and $n_{r m}$ are the reference and the measured motor speed. In Fig. 18, the M3C passes through three critical operation points at $f_{2}=50 \mathrm{~Hz}, f_{2}=0$ and $f_{2}=-50 \mathrm{~Hz}$. Capacitor-voltages shown in Fig. 18 (d) prove the effectiveness of the proposed balancing control. The magnitude of the injected CMV in Fig. 18 (f) coincides with the limitation in Fig. 7. As the maximum value of the circulating current $I_{c i r, M A X}$ in (22) is set as $2 \mathrm{~A}$, there is no obvious increase on branch current magnitude passing through critical-frequency points in Fig. 18 (e).
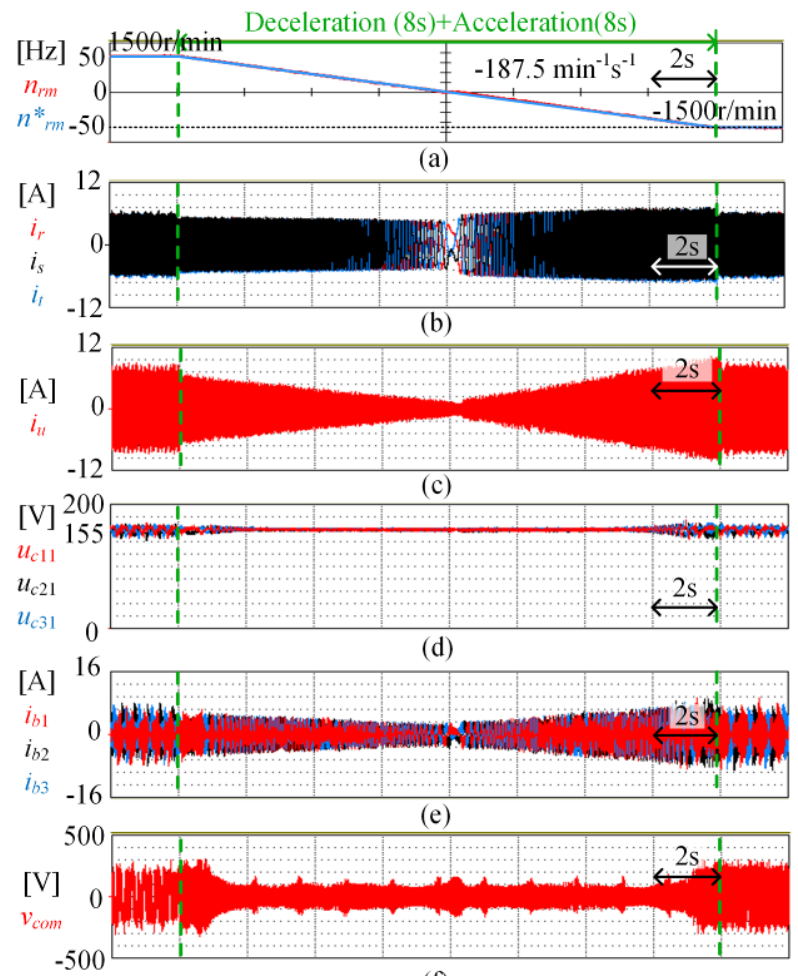

(f)

Fig. 18. The deceleration and acceleration experiment $(1500 \mathrm{r} / \mathrm{min}$ to $-1500 \mathrm{r} / \mathrm{min}$, constant $60 \%$ rated torque). (a) Motor speed. (b) Output currents. (c) The input current on phase-u. (d) Capacitor voltages. (e) Branch currents. (f) The CMV.

Fig. 19 shows the comparison of the theoretically calculated capacitor-voltage fluctuation without voltage-balancing control and the measured capacitor-voltage fluctuation with proposed control. In Fig. 19, the theoretical capacitor-voltage fluctuation, shown in blue, is calculated according to (8). The measured capacitor-voltage fluctuation is shown in red triangular. The red solid line is the theoretically calculated output frequency, which is larger than the motor speed $(\mathrm{Hz})$ by a slip frequency. In Fig. 19, when the output frequency gets closer to the AC grid frequency $\left(f_{2} \approx 50 \mathrm{~Hz}\right)$, theoretically, there will be large capacitor fluctuations but with the proposed control, the capacitor-voltage fluctuation is effectively suppressed. When 
the output frequency is away from $50 \mathrm{~Hz}$, as explained in Section III-D, the injected CMV and circulating currents are limited according to (31) to compensate only small branch power differences caused by modulation or calculation inaccuracy. In this condition, the circulating current and CMV injection will not influence the capacitor-voltage fluctuation. As shown in the Fig. 19, when the output frequency is smaller than $40 \mathrm{~Hz}$, the theoretical capacitor-voltage fluctuation coincides with the measured capacitor-voltage fluctuation very well.

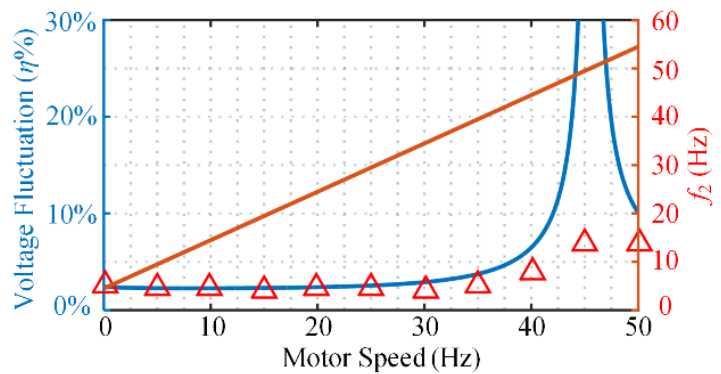

Fig. 19. Verification of the capacitor-voltage fluctuation (Induction motor load, $60 \%$ rated torque)

\section{CONCLUSIONS}

In this paper, an optimization based predictive control method is proposed for the M3C. This paper firstly analyzed the basic branch power with no CMV and circulating current injected. When the $\mathrm{M} 3 \mathrm{C}$ is operated away from critical-frequency points, balancing control only needs to compensate small branch power imbalances caused by the modulation or small calculation inaccuracy. When the M3C is operated at or around critical-frequency points, the CMV and circulating currents are required to compensate the $\mathrm{dc}$ or extremely low frequency branch powers that cause large capacitor-voltage fluctuation.

In the proposed control, the injection of CMV is first optimized. Then based on the optimized CMV, a sub-optimized solution of the circulating currents is designed to further reduce the capacitor-voltage fluctuation. Compared with the existing control method, the main improvement of the proposed control is that it fully utilizes the available range and bandwidth of the CMV and circulating currents so that the branch energy can be balanced among nine branches efficiently. The proposed new method features a broad frequency range balancing of capacitor-voltages and ensures a unity factor at $\mathrm{AC}$ grid at any frequency operation. For operations at critical-frequency points, there is no increase on branch voltage stresses and only limited increase on branch current stresses. Besides, the proposed control can be easily extended to many other modular cascaded topologies such as the MMC and the hexagonal modular multilevel converter (HMMC). To reduce the possible damage on motor bearings and to increase system efficiency, the magnitudes of the CMV and circulating currents have been limited according to the operation frequency. The theoretical analysis and proposed control strategy are validated by experiment results with R-L load and induction motor load. Moreover, it is worthy to mention that the proposed control can help the M3C pass through or work at critical-frequency points but it is recommended not making the $\mathrm{M} 3 \mathrm{C}$ continuously work under these conditions because a relatively large injection of CMV and circulating currents is inevitable.

\section{REFERENCES}

[1] H. Akagi, "Classification, Terminology, and Application of the Modular Multilevel Cascade Converter (MMCC)," IEEE Transactions on Power Electronics, vol. 26, no. 11. pp. 3119-3130, 2011.

[2] R. W. Erickson and O. A. Al-Naseem, "A new family of matrix converters," Industrial Electronics Society, 2001. IECON '01. The 27th Annual Conference of the IEEE, vol. 2. pp. 1515-1520 vol.2, 2001.

[3] S. Angkititrakul and R. W. Erickson, "Control and implementation of a new modular matrix converter," Applied Power Electronics Conference and Exposition, 2004. APEC '04. Nineteenth Annual IEEE, vol. 2. pp. 813-819 vol.2, 2004.

[4] S. Angkititrakul and R. W. Erickson, "Capacitor voltage balancing control for a modular matrix converter," Twenty-First Annual IEEE Applied Power Electronics Conference and Exposition, 2006. APEC '06. p. 7 pp., 2006.

[5] Y. Li, L. Xu, and Y. Li, "A novel modulation with voltage balancing control for a modular matrix converter," 2014 17th International Conference on Electrical Machines and Systems (ICEMS). pp. 2483-2489, 2014

[6] C. Oates, "A Methodology for Developing 'Chainlink' Converters," Power Electronics and Applications, 2009. EPE '09. 13th European Conference on. pp. 1-10, 2009.

[7] F. Kammerer, J. Kolb, and M. Braun, "Fully decoupled current control and energy balancing of the Modular Multilevel Matrix Converter," Power Electronics and Motion Control Conference (EPE/PEMC), 2012 15th International. p. LS2a.3-1-LS2a.3-8, 2012.

[8] W. Kawamura and H. Akagi, "Control of the modular multilevel cascade converter based on triple-star bridge-cells (MMCC-TSBC) for motor drives," 2012 IEEE Energy Conversion Congress and Exposition (ECCE). pp. 3506-3513, 2012.

[9] W. Kawamura, M. Hagiwara, and H. Akagi, "Control and Experiment of a Modular Multilevel Cascade Converter Based on Triple-Star Bridge Cells (MMCC-TSBC)," IEEE Transactions on Industry Applications, vol. 50, no. 5. pp. 3536-3548, 2014.

[10] W. Kawamura, M. Hagiwara, and H. Akagi, "Control and experiment of a $380-\mathrm{V}, 15-\mathrm{kW}$ motor drive using modular multilevel cascade converter based on triple-star bridge cells (MMCC-TSBC)," 2014 International Power Electronics Conference (IPEC-Hiroshima 2014 - ECCE ASIA). pp. 3742-3749, 2014.

[11] K. Wang, Y. Li, Z. Zheng, and L. Xu, "Voltage Balancing and Fluctuation-Suppression Methods of Floating Capacitors in a New Modular Multilevel Converter," IEEE Transactions on Industrial Electronics, vol. 60, no. 5. pp. 1943-1954, 2013.

[12] J. Kolb, F. Kammerer, M. Gommeringer, and M. Braun, "Cascaded Control System of the Modular Multilevel Converter for Feeding Variable-Speed Drives," IEEE Transactions on Power Electronics, vol. 30, no. 1. pp. 349-357, 2015.

[13] A. Antonopoulos, L. Angquist, S. Norrga, K. Ilves, L. Harnefors, and H. P. Nee, "Modular Multilevel Converter AC Motor Drives With Constant Torque From Zero to Nominal Speed," IEEE Transactions on Industry Applications, vol. 50, no. 3. pp. 1982-1993, 2014.

[14] F. Kammerer, D. Braeckle, M. Gommeringer, M. Schnarrenberger, and M. Braun, "Operating Performance of the Modular Multilevel Matrix Converter in Drive Applications," PCIM Europe 2015; International Exhibition and Conference for Power Electronics, Intelligent Motion, Renewable Energy and Energy Management; Proceedings of. pp. 1-8, 2015.

[15] K. Ilves, L. Bessegato, and S. Norrga, "Comparison of cascaded multilevel converter topologies for AC/AC conversion," 2014 International Power Electronics Conference (IPEC-Hiroshima 2014 ECCE ASIA). pp. 1087-1094, 2014.

[16] Y. Okazaki et al., "Experimental Comparisons Between Modular Multilevel DSCC Inverters and TSBC Converters for Medium-Voltage Motor Drives," IEEE Transactions on Power Electronics, vol. 32, no. 3. pp. 1805-1817, 2017.

[17] M. Diaz, R. Cardenas, B. M. Espinoza, A. Mora, and F. Rojas, "A novel LVRT control strategy for Modular Multilevel Matrix Converter based high-power Wind Energy Conversion Systems," Ecological Vehicles 
and Renewable Energies (EVER), 2015 Tenth International Conference on. pp. 1-11, 2015.

[18] J. M. Apsley et al., "Propulsion Drive Models for Full Electric Marine Propulsion Systems," IEEE Transactions on Industry Applications, vol. 45, no. 2. pp. 676-684, 2009.

[19] S. Liu, X. Wang, Y. Meng, P. Sun, H. Luo, and B. Wang, "A Decoupled Control Strategy of Modular Multilevel Matrix Converter for Fractional Frequency Transmission System," IEEE Transactions on Power Delivery, vol. PP, no. 99. p. 1, 2016.

[20] Y. Miura, T. Mizutani, M. Ito, and T. Ise, "Modular multilevel matrix converter for low frequency AC transmission," Power Electronics and Drive Systems (PEDS), 2013 IEEE 10th International Conference on. pp. 1079-1084, 2013.

[21] B. Fan, K. Wang, P. Wheeler, C. Gu, and Y. Li, "A Branch Current Reallocation Based Energy Balancing Strategy for the Modular Multilevel Matrix Converter Operating Around Equal Frequency," IEEE Transactions on Power Electronics, vol. PP, no. 99. p. 1, 2017.

[22] A. J. Korn, M. Winkelnkemper, P. Steimer, and J. W. Kolar, "Direct modular multi-level converter for gearless low-speed drives," Power Electronics and Applications (EPE 2011), Proceedings of the 2011-14th European Conference on. pp. 1-7, 2011.

[23] W. Kawamura, M. Hagiwara, and H. Akagi, "A broad range of frequency control for the modular multilevel cascade converter based on triple-star bridge-cells (MMCC-TSBC)," 2013 IEEE Energy Conversion Congress and Exposition. pp. 4014-4021, 2013.

[24] W. Kawamura, Y. Chiba, M. Hagiwara, and H. Akagi, "Experimental Verification of an Electrical Drive Fed by a Modular Multilevel TSBC Converter When the Motor Frequency Gets Closer or Equal to the Supply Frequency," IEEE Transactions on Industry Applications, vol. PP, no. 99. p. 1, 2017.

[25] F. Kammerer, M. Gommeringer, J. Kolb, and M. Braun, "Energy balancing of the Modular Multilevel Matrix Converter based on a new transformed arm power analysis," Power Electronics and Applications (EPE'14-ECCE Europe), 2014 16th European Conference on. pp. 1-10, 2014.

[26] W. Kawamura, Y. Chiba, and H. Akagi, "A broad range of speed control of a permanent magnet synchronous motor driven by a modular multilevel TSBC converter," 2016 IEEE Energy Conversion Congress and Exposition (ECCE). pp. 1-6, 2016.

[27] F. Kammerer, J. Kolb, and M. Braun, "A novel cascaded vector control scheme for the Modular Multilevel Matrix Converter," IECON 2011 37th Annual Conference on IEEE Industrial Electronics Society. pp. 1097-1102, 2011.

[28] M. Hagiwara and H. Akagi, "Control and Experiment of Pulsewidth-Modulated Modular Multilevel Converters," IEEE Transactions on Power Electronics, vol. 24, no. 7. pp. 1737-1746, 2009.

[29] M. P. Kazmierkowski and L. Malesani, "Current control techniques for three-phase voltage-source PWM converters: a survey," IEEE Transactions on Industrial Electronics, vol. 45, no. 5. pp. 691-703, 1998.

[30] B. Fan, K. Wang, C. Gu, P. Wheeler, and Y. Li, "A branch current reallocation based energy balancing strategy for the Modular multilevel matrix converter operating around equal frequency," IECON 2016 42nd Annual Conference of the IEEE Industrial Electronics Society. pp. 3111-3116, 2016.

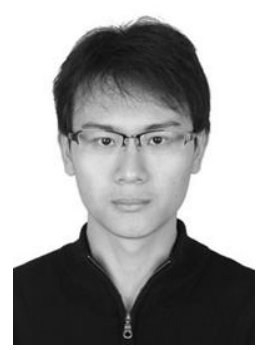

Boran Fan ( $\mathrm{S}^{\prime}$ 13) was born in Tianjin, China, in 1989. He received the B.S. degree in Electrical Engineering from the Department of Electrical Engineering, Tsinghua University, Beijing, China, in 2013. He is currently working toward the Ph.D. degree with the Department of Electrical Engineering, Tsinghua University. His current research interests are topology and control of multilevel converters.

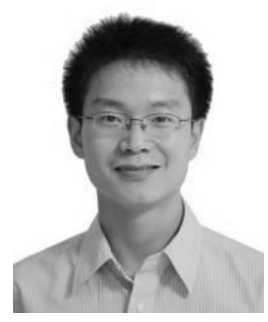

Kui Wang (M'11) was born in Hubei, China, in 1984. He received the B.S. and Ph.D. degrees in Electrical Engineering from the Department of Electrical Engineering, Tsinghua University, Beijing, China, in 2006 and 2011, respectively. He is currently a Faculty Member with the Department of Electrical Engineering, Tsinghua University. His research interests are topology and control of multilevel converters, renewable energy generation and wide band-gap semiconductor.

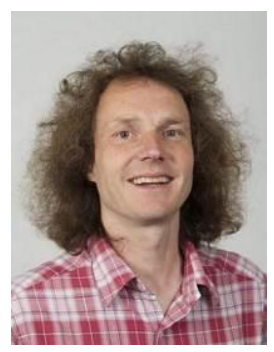

Pat Wheeler (SM'11) received his BEng [Hons] degree in 1990 from the University of Bristol, UK. $\mathrm{He}$ received his $\mathrm{PhD}$ degree in Electrical Engineering for his work on Matrix Converters from the University of Bristol, UK in 1994. In 1993 he moved to the University of Nottingham and worked as a research assistant in the Department of Electrical and Electronic Engineering. In 1996 he became a Lecturer in the Power Electronics, Machines and Control Group at the University of Nottingham, UK. Since January 2008 he has been a Full Professor in the same research group. He is currently Head of the Department of Electrical and Electronic Engineering at the University of Nottingham. He is an IEEE PELs 'Member at Large' and an IEEE PELs Distinguished Lecturer. $\mathrm{He}$ has published 400 academic publications in leading international conferences and journals.

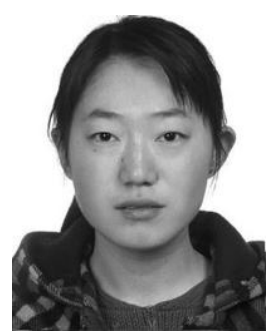

Chunyang Gu (S'12-M'15) was born in Heilongjiang, China, in 1988. She received the B.Sc. degree in Electrical Engineering from Harbin Institute of Technology, Harbin, China, in 2010 She received Ph.D. degree in Electrical Engineering from Department of Electrical Engineering, Tsinghua University, Beijing, China, in 2015. She is currently a Research Fellow in Power Electronics, Machines and Control (PEMC) Research Group, The University of Nottingham, Nottingham, U.K. Her current research interests include solid-state circuit breakers, power converter topologies and control, multilevel power converters, solid-state transformers, and application of WBG semiconductor devices.

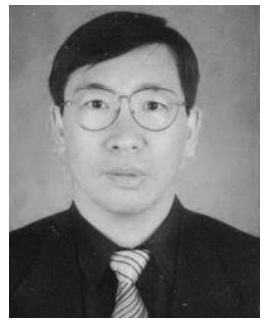

Yongdong Li (M'08) was born in Hebei, China, in 1962. He received the B.S. degree from Harbin Institute of Technology, China, in 1982 and the M.S. and Ph.D. degrees from the Department of Electrical Engineering, Institut National Polytechnique de Toulouse, Toulouse, France, in 1984 and 1987, respectively. Since 1996, he has been a Professor with the Department of Electrical Engineering, Tsinghua University, Beijing, China. His research interests include power electronics, machine control, and wind power generation. 